\title{
CHEMICAL ABUNDANCES IN THE EXTREMELY CARBON AND XENON-RICH HALO PLANETARY NEBULA H4-1
}

\author{
MASAAKI OTSUKA ${ }^{1}$ AND AKITO TAJITSU ${ }^{2}$ \\ ${ }^{1}$ Institute of Astronomy and Astrophysics, Academia Sinica P.O. Box 23-141, Taipei 10617, Taiwan, Republic of China; otsuka@asiaa.sinica.edu.tw and \\ ${ }^{2}$ Subaru Telescope, NAOJ, 650 North A'ohoku Place, Hilo, HI 96720, U.S.A.; tajitsu@ subaru.naoj.org
}

(Received; Revised; Accepted)

\begin{abstract}
We performed detailed chemical abundance analysis of the extremely metal-poor $([\mathrm{Ar} / \mathrm{H}] \sim-2)$ halo planetary nebula H4-1 based on the multi-wavelength spectra from Subaru/HDS, GALEX, SDSS, and Spitzer/IRS and determined the abundances of 10 elements. The $\mathrm{C}$ and $\mathrm{O}$ abundances were derived from collisionally excited lines (CELs) and are almost consistent with abundances from recombination lines (RLs). We demonstrated that the large discrepancy in the $\mathrm{C}$ abundance between CEL and RL in H4-1 can be solved using the temperature fluctuation model. We reported the first detection of the [Xe III] $\lambda 5846 \AA$ line in $\mathrm{H} 4-1$ and determination of its elemental abundance $([\mathrm{Xe} / \mathrm{H}]>+0.48)$. H4-1 is the most Xe-rich PN among the Xe-detected PNe. The observed abundances are close to the theoretical prediction by a $\sim 2.0 M_{\odot}$ single star model with initially $r$ process element rich $([r / \mathrm{Fe}]=+2.0 \mathrm{dex})$. The observed Xe abundance would be a product of the $r$-process in primordial $\mathrm{SNe}$. The $[\mathrm{C} / \mathrm{O}]-[\mathrm{Ba} /(\mathrm{Eu}$ or $\mathrm{Xe})]$ diagram suggests that the progenitor of $\mathrm{H} 4-1$ shares the evolution with two types of carbon-enhanced metal-poor stars (CEMP), CEMP-r/s and CEMP-no stars. The progenitor of H4-1 is a presumably binary formed in an $r$-process rich environment.
\end{abstract}

Subject headings: ISM: planetary nebulae: individual (H4-1), ISM: abundances

\section{INTRODUCTION}

Currently, more than 1000 objects are considered planetary nebulae (PNe) in the Galaxy, about 14 of which have been identified as halo members because of their current location and kinematics (e.g., Otsuka et al. 2008a). Halo PNe are interesting objects because they provide direct insight into the final evolution of old, low-mass metal-poor halo stars.

The halo PN H4-1 is extremely metal-poor $\left(Z \sim 10^{-4}\right.$, $[\mathrm{Fe} / \mathrm{H}]=-2.3)$ and C-rich (Torres-Peimbert \& Peimbert 1979; Kwitter et al. 2003), similar to the C-rich halo PNe BoBn1 in the Sagittarius dwarf spheroidal galaxy (Otsuka et al. 2010, 2008a) and K648 in M15 (Rauch et al. 2002). The chemical abundances and metallicity of these three PNe imply that their progenitors formed early, perhaps $\sim 10-13$ Gyrs ago. However, these extremely metal-poor halo PNe have an unresolved issue: how did their progenitors evolve into C-rich $\mathrm{PNe}$ ?

The initial mass of these three PNe is generally thought to equal $\sim 0.8 M_{\odot}$, which corresponds to the turn-off stellar mass of M15. According to recent stellar evolution models (e.g., Lugaro et al.2012; Fujimoto et al. 2000), there are two mechanisms for stars with $Z \lesssim 10^{-4}$ to become C-rich: (1) heliumflash driven deep mixing (He-FDDM) for $<2.5-3 M_{\odot}$ stars and (2) third dredge-up (TDU) for $\gtrsim 0.9-1.5 M_{\odot}$ stars. Mechanism (1) is unlikely for these PNe because this mechanism occurs in stars with $Z \lesssim 6 \times 10^{-5}([\mathrm{Fe} / \mathrm{H}] \lesssim-2.5)$. Therefore, TDU is essential for these PNe to become C-rich, implying that it is possible that these halo PNe evolved from $\gtrsim 0.8-0.9$ $M_{\odot}$ single stars. We should note that the lower limit mass required for TDU depends largely on models. The minimum mass for the occurrence of the TDU is thought to be 1.2-1.5 $M_{\odot}$ (e.g., Lattanzio 1987; Boothroyd \& Sackmann 1988).

Even if the progenitor of $\mathrm{H} 4-1$ is a $\gtrsim 0.8-0.9 M_{\odot}$ single star and it experienced TDU during AGB phase, current low-mass stellar evolution models are unlikely to explain the evolution of H4-1; the effective temperature of the central star of H4-1 is between 93400 (Mal'Kov 1997) and $132000 \mathrm{~K}$ (Henry et al. 1996), and the age of the PN is 8400 yrs, assuming a dis- tance of $25.3 \mathrm{kpc}$ from us (Mal'Kov 1997). The He-shell burning model for the initially $0.89 M_{\odot}$ and $Z=0.004$ stars by Vassiliadis \& Wood (1994) predicted that it takes $>50000$ yrs for a star to reach $10^{5} \mathrm{~K}$. There is obviously a large discrepancy in evolutional time scale between the observations and theoretical models.

To explain the evolution of H4-1, we need to consider additional mechanisms that shorten the evolutionary timescale, e.g., binary mass-transfer via Roche lobe overflow, which was proposed for BoBn1 by Otsuka et al. (2010). Evidence suggests that binary evolution is the most plausible scenario for H4-1. In particular, Tajitsu \& Otsuka (2004) showed that H4-1 has a bright equatorial disk, a bipolar nebula, and multiple arcs in its molecular hydrogen image. The discovery of these structures implies that H4-1 would have evolved from a binary, similar to carbon-enhanced metalpoor (CEMP) stars found in the Galactic halo and these CEMP stars show $[\mathrm{Fe} / \mathrm{H}]<-2$ (see Beers \& Christlieb 2005). Otsuka et al. (2008b) found that the location of H4-1 on $[\mathrm{C} /(\mathrm{Fe}$ or $\mathrm{Ar})]$ versus $[(\mathrm{Fe}$ or $\mathrm{Ar}) / \mathrm{H}]$ diagrams is in the region occupied by CEMP stars, indicating a similar origin and evolution.

Elements with atomic number $Z>30$ are synthesized in the neutron $(n)$ capturing process in both $\mathrm{PN}$ progenitors and supernovae (SNe). In PN progenitors, the $n$-flux is much lower, so the $n$-capturing process is a slow process ( $s$-process). In $\mathrm{SNe}$, because of a much higher $n$-flux, the $n$-capturing process is a rapid process ( $r$-process). Several types of CEMP stars show the enhancements of $s$ - and/or $r$-process elements. Therefore, if we can detect any $n$-capture elements, we would be able to obtain important insights into the evolution of H4-1 and the chemical environment where its progenitor was born by comparing the carbon and $n$-capture elements of H4- 1 and those of CEMP stars.

The elements $\mathrm{C}$ and $\mathrm{O}$ are clearly important with respect to the evolution of H4-1. However, there is a large discrepancy in the $\mathrm{C}$ abundances between collisionally excited lines (CELs) and recombination lines (RLs). 


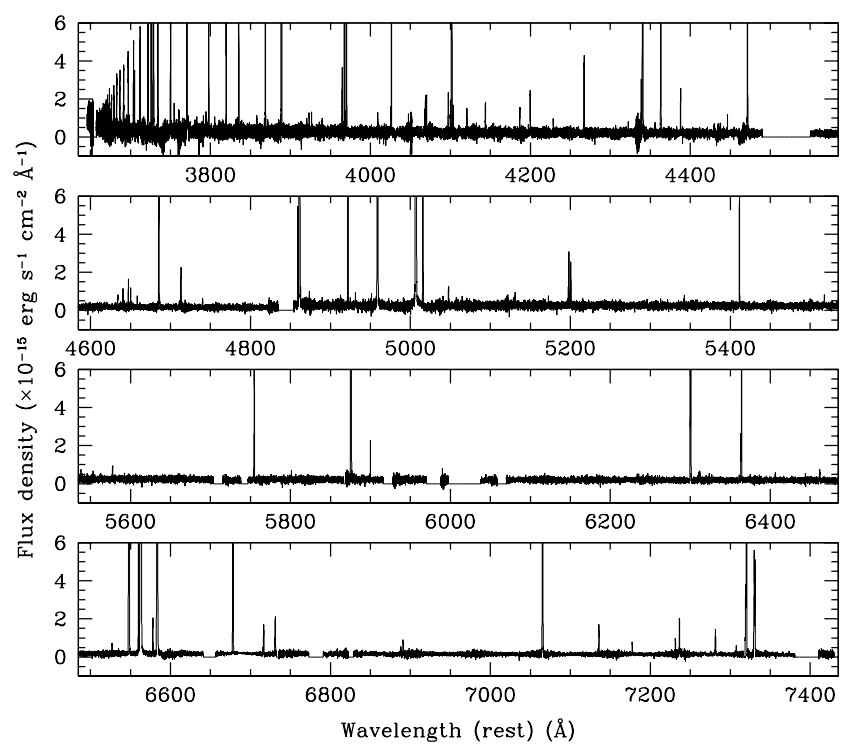

FIG. 1.- The de-reddened HDS spectrum of H4-1. The wavelength is shifted to the rest wavelength in air.

Torres-Peimbert \& Peimbert (1979) estimated the C number density using RLs and found that $\log n(\mathrm{C}) / n(\mathrm{H})+12=9.39$, whereas Henry et al. (1996) found a value of 8.68 using CELs. Discrepancies in $\mathrm{C}$ and $\mathrm{O}$ abundances have already been found in many Galactic PNe (e.g., Liu 2006). It is necessary to solve the $\mathrm{C}$ discrepancy in $\mathrm{H} 4-1$ (the $\mathrm{O}$ discrepancy, too if exist).

In this paper, we report a detail chemical abundance analysis of H4-1 based on deep high-dispersion optical spectra from Subaru/HDS, and archived UV, optical, and mid-IR spectra from GALEX, the Sloan Digital Sky Survey (SDSS), and Spitzer/IRS, respectively. In Section 2, we describe the observations. In Section 3, we provide the ionic and elemental abundances of H4-1 derived from CELs and RLs. The first detection of the $n$-capture element xenon (Xe) is reported in this section. In Section 4.1, we discuss the discrepancy in $\mathrm{C}$ and $\mathrm{O}$ abundances. In Sections 4.2 and 4.3, we discuss the Xe abundance by comparing with the theoretical nucleosynthesis model and the origin and evolution of H4-1 from the view point of chemical abundances. A summary is given in Section 5 .

\section{OBSERVATIONS \& DATA REDUCTION}

\subsection{HDS observations}

The spectra of H4-1 were taken using the High-Dispersion Spectrograph (HDS; Noguchi et al. 2002) attached to one of the two Nasmyth foci of the 8.2-m Subaru telescope (Program ID: S09A-163S, PI: M.Otsuka).

The red spectra (4600-7500 ̊) were obtained in May 2009, when weather conditions were unfavorable; there were scattered cirrus clouds in the sky and a full moon. The seeing was $\sim 1.5^{\prime \prime}$ from the guider CCD. An atmospheric dispersion corrector (ADC) was used to minimize the differential atmospheric dispersion through the broad wavelength region. We set the slit width to equal $1 . \prime 5$ and chose a $2 \times 2$ on-chip binning. We set the slit length to $7^{\prime \prime}$, which fit the nebula and allowed a direct subtraction of sky background from the object frames. The CCD sampling pitch along the slit length projected on the sky equaled $\sim 0$." 276 per a binned pixel. The resolving power reached approximately $R>33000$, which is derived from the mean full width at half maximum (FWHM)
TABLE 1

DETECTED LINES AND IDENTIFICATIONS OF H4-1 IN HDS SPECTRA.

\begin{tabular}{|c|c|c|c|c|c|c|}
\hline $\begin{array}{l}\lambda_{\text {obs }} \\
(\AA)\end{array}$ & Ion & $\begin{array}{l}\lambda_{\text {lab }} \\
(\AA)\end{array}$ & Comp. & $f(\lambda)$ & $I(\lambda)$ & $\delta I(\lambda)$ \\
\hline 3667.25 & $\mathrm{H} 25$ & 3669.46 & 1 & 0.334 & 0.355 & 0.047 \\
\hline 3669.30 & $\mathrm{H} 24$ & 3671.48 & 1 & 0.333 & 0.386 & 0.058 \\
\hline 3671.53 & $\mathrm{H} 23$ & 3673.74 & 1 & 0.333 & 0.439 & 0.096 \\
\hline 3672.35 & He II & 3674.84 & 1 & 0.333 & 0.177 & 0.054 \\
\hline 3674.11 & $\mathrm{H} 22$ & 3676.36 & 1 & 0.332 & 0.584 & 0.066 \\
\hline 3677.16 & $\mathrm{H} 21$ & 3679.35 & 1 & 0.332 & 0.564 & 0.061 \\
\hline 3680.61 & $\mathrm{H} 20$ & 3682.81 & 1 & 0.331 & 0.672 & 0.052 \\
\hline 3684.64 & H19 & 3686.83 & 1 & 0.330 & 0.906 & 0.069 \\
\hline 3689.34 & H18 & 3691.55 & 1 & 0.329 & 0.911 & 0.062 \\
\hline 3694.92 & H17 & 3697.15 & 1 & 0.328 & 1.175 & 0.075 \\
\hline 3701.62 & H16 & 3703.65 & 1 & 0.327 & 1.316 & 0.073 \\
\hline 3702.81 & He I & 3704.98 & 1 & 0.327 & 0.647 & 0.047 \\
\hline 3704.95 & O III & 3707.25 & 1 & 0.326 & 0.056 & 0.038 \\
\hline 3709.73 & H15 & 3711.97 & 1 & 0.325 & 1.513 & 0.075 \\
\hline 3712.80 & O III & 3715.08 & 1 & 0.325 & 0.085 & 0.028 \\
\hline 3719.69 & H14 & 3721.94 & 1 & 0.323 & 1.971 & 0.090 \\
\hline 3723.63 & [O II] & 3726.03 & 1 & 0.322 & 50.749 & 2.492 \\
\hline \multirow[t]{2}{*}{3723.94} & [O II] & 3726.03 & 2 & 0.322 & 40.319 & 2.203 \\
\hline & & & Tot. & & 91.068 & 3.326 \\
\hline 3726.33 & [O II] & 3728.81 & 1 & 0.322 & 42.426 & 1.851 \\
\hline \multirow[t]{2}{*}{3726.69} & [O II] & 3728.81 & 2 & 0.322 & 42.795 & 1.841 \\
\hline & & & Tot. & & 85.221 & 2.610 \\
\hline 3730.62 & $\mathrm{He} \mathrm{I}$ & 3732.86 & 1 & 0.321 & 0.103 & 0.031 \\
\hline 3732.12 & H13 & 3734.37 & 1 & 0.321 & 2.465 & 0.109 \\
\hline 3747.89 & H12 & 3750.15 & 1 & 0.317 & 2.991 & 0.125 \\
\hline 3752.40 & O III & 3754.70 & 1 & 0.316 & 0.182 & 0.020 \\
\hline 3768.37 & H11 & 3770.63 & 1 & 0.313 & 3.775 & 0.155 \\
\hline 3795.61 & $\mathrm{H} 10$ & 3797.90 & 1 & 0.307 & 4.840 & 0.194 \\
\hline 3817.34 & He I & 3819.60 & 1 & 0.302 & 1.238 & 0.054 \\
\hline 3831.40 & He I & 3833.55 & 1 & 0.299 & 0.128 & 0.030 \\
\hline 3833.08 & H9 & 3835.38 & 1 & 0.299 & 7.301 & 0.286 \\
\hline 3855.71 & He II & 3858.07 & 1 & 0.294 & 0.105 & 0.024 \\
\hline 3865.16 & He I & 3867.47 & 1 & 0.291 & 0.136 & 0.021 \\
\hline 3866.44 & [Ne III] & 3868.77 & 1 & 0.291 & 5.323 & 0.225 \\
\hline 3869.43 & He I & 3871.83 & 1 & 0.290 & 0.091 & 0.020 \\
\hline 3886.73 & $\mathrm{H} 8$ & 3889.05 & 1 & 0.286 & 9.712 & 0.387 \\
\hline 3918.27 & C II & 3920.68 & 1 & 0.279 & 0.050 & 0.018 \\
\hline 3921.09 & He II & 3923.48 & 1 & 0.278 & 0.124 & 0.016 \\
\hline 3924.18 & $\mathrm{He} \mathrm{I}$ & 3926.54 & 1 & 0.277 & 0.111 & 0.014 \\
\hline 3962.36 & He I & 3964.73 & 1 & 0.267 & 0.658 & 0.036 \\
\hline 3965.08 & [Ne III] & 3967.46 & 1 & 0.267 & 1.562 & 0.059 \\
\hline 3966.01 & He II & 3968.43 & 1 & 0.266 & 0.170 & 0.020 \\
\hline 3967.68 & $\mathrm{H} 7$ & 3970.07 & 1 & 0.266 & 14.235 & 0.506 \\
\hline 4006.90 & He I & 4009.26 & 1 & 0.256 & 0.191 & 0.021 \\
\hline 4023.81 & He I & 4026.18 & 1 & 0.251 & 1.969 & 0.068 \\
\hline 4065.47 & $\mathrm{C}_{\text {III }}$ & 4067.87 & 1 & 0.239 & 0.162 & 0.015 \\
\hline 4066.40 & [S II] & 4068.60 & 1 & 0.239 & 0.277 & 0.019 \\
\hline 4067.77 & O II & 4070.14 & 1 & 0.239 & 0.261 & 0.018 \\
\hline 4089.64 & O II & 4092.93 & 1 & 0.232 & 0.043 & 0.019 \\
\hline 4094.83 & N III & 4097.35 & 1 & 0.231 & 0.283 & 0.016 \\
\hline 4097.54 & He II & 4100.04 & 1 & 0.230 & 0.249 & 0.039 \\
\hline 4099.27 & H6 & 4101.73 & 1 & 0.230 & 25.395 & 0.781 \\
\hline 4100.90 & N III & 4103.39 & 1 & 0.229 & 0.231 & 0.028 \\
\hline 4118.37 & He I & 4120.81 & 1 & 0.224 & 0.269 & 0.026 \\
\hline 4126.21 & [Fe II] & 4128.75 & 1 & 0.222 & 0.077 & 0.024 \\
\hline 4141.30 & $\mathrm{He} \mathrm{I}$ & 4143.76 & 1 & 0.217 & 0.320 & 0.020 \\
\hline 4197.27 & He II & 4199.83 & 1 & 0.200 & 0.378 & 0.014 \\
\hline 4264.60 & C II & 4267.15 & 1 & 0.180 & 1.118 & 0.035 \\
\hline 4336.02 & He II & 4338.67 & 1 & 0.157 & 0.393 & 0.070 \\
\hline 4337.85 & H5 & 4340.46 & 1 & 0.157 & 46.910 & 1.069 \\
\hline 4346.71 & O II & 4349.43 & 1 & 0.154 & 0.028 & 0.006 \\
\hline 4360.52 & [O III] & 4363.21 & 1 & 0.149 & 3.677 & 0.167 \\
\hline \multirow[t]{2}{*}{4360.58} & [O III] & 4363.21 & 2 & 0.149 & 6.078 & 0.159 \\
\hline & & & Tot. & & 9.755 & 0.231 \\
\hline 4385.32 & He I & 4387.93 & 1 & 0.142 & 0.532 & 0.016 \\
\hline 4387.89 & C III & 4390.50 & 1 & 0.141 & 0.071 & 0.020 \\
\hline 4434.94 & He I & 4437.55 & 1 & 0.126 & 0.083 & 0.017 \\
\hline 4468.83 & He I & 4471.47 & 1 & 0.115 & 4.487 & 0.093 \\
\hline 4558.82 & Mg I] & 4562.60 & 1 & 0.087 & 0.031 & 0.007 \\
\hline 4631.33 & N III & 4634.12 & 1 & 0.065 & 0.083 & 0.011 \\
\hline 4635.99 & O II & 4638.86 & 1 & 0.064 & 0.057 & 0.006 \\
\hline 4637.80 & N III & 4640.64 & 1 & 0.063 & 0.148 & 0.006 \\
\hline 4639.03 & O II & 4641.81 & 1 & 0.063 & 0.047 & 0.005 \\
\hline 4644.57 & C III & 4647.42 & 1 & 0.061 & 0.232 & 0.007 \\
\hline
\end{tabular}


TABLE 1

CONTINUED.

\begin{tabular}{|c|c|c|c|c|c|c|}
\hline $\begin{array}{c}\lambda_{\text {obs }} \\
(\AA)\end{array}$ & Ion & $\begin{array}{l}\lambda_{\text {lab }} \\
(\AA)\end{array}$ & Comp. & $f(\lambda)$ & $I(\lambda)$ & $\delta I(\lambda)$ \\
\hline 4647.39 & C III & 4650.25 & 1 & 0.060 & 0.147 & 0.006 \\
\hline 4648.66 & O II & 4651.33 & 1 & 0.060 & 0.024 & 0.005 \\
\hline 4655.58 & CIV & 4658.20 & 1 & 0.058 & 0.065 & 0.004 \\
\hline 4658.89 & O II & 4661.63 & 1 & 0.057 & 0.035 & 0.005 \\
\hline 4682.60 & He II & 4685.68 & 1 & 0.050 & 4.491 & 0.109 \\
\hline \multirow[t]{2}{*}{4682.91} & He II & 4685.68 & 2 & 0.050 & 13.819 & 0.199 \\
\hline & & & Tot. & & 18.310 & 0.227 \\
\hline 4708.49 & [Ar IV] & 4711.37 & 1 & 0.042 & 0.065 & 0.007 \\
\hline 4710.36 & $\mathrm{He} \mathrm{I}$ & 4713.17 & 1 & 0.042 & 0.554 & 0.013 \\
\hline 4737.32 & [Ar IV] & 4740.17 & 1 & 0.034 & 0.059 & 0.004 \\
\hline 4856.37 & He II & 4859.32 & 1 & 0.001 & 0.873 & 0.017 \\
\hline 4858.37 & H4 & 4861.33 & 1 & 0.000 & 42.807 & 1.844 \\
\hline \multirow[t]{2}{*}{4858.43} & $\mathrm{H} 4$ & 4861.33 & 2 & 0.000 & 57.193 & 1.731 \\
\hline & & & Tot. & & 100.000 & 2.529 \\
\hline 4919.00 & $\mathrm{He} \mathrm{I}$ & 4921.93 & 1 & -0.016 & 1.006 & 0.017 \\
\hline 4928.29 & [O III] & 4931.80 & 1 & -0.019 & 0.073 & 0.013 \\
\hline 4955.89 & [O III] & 4958.91 & 1 & -0.026 & 112.614 & 2.513 \\
\hline \multirow[t]{2}{*}{4955.96} & [O III $]$ & 4958.91 & 2 & -0.026 & 111.463 & 1.811 \\
\hline & & & Tot. & & 224.077 & 3.097 \\
\hline 5003.67 & [O III] & 5006.84 & 1 & -0.038 & 76.548 & 18.101 \\
\hline 5003.85 & [O III] & 5006.84 & 2 & -0.038 & 578.884 & 17.282 \\
\hline \multirow{2}{*}{5004.51} & [O III $]$ & 5006.84 & 3 & -0.038 & 12.015 & 7.893 \\
\hline & & & Tot. & & 667.447 & 26.242 \\
\hline 5012.69 & $\mathrm{He} \mathrm{I}$ & 5015.68 & 1 & -0.040 & 1.907 & 0.035 \\
\hline 5028.99 & C II & 5032.07 & 1 & -0.044 & 0.044 & 0.008 \\
\hline 5032.83 & {$[\mathrm{Fe} \mathrm{II}]$} & 5035.48 & 1 & -0.045 & 0.030 & 0.005 \\
\hline 5044.74 & $\mathrm{He} \mathrm{I}$ & 5047.74 & 1 & -0.048 & 0.153 & 0.007 \\
\hline 5124.28 & $\mathrm{Fe}$ III & 5127.39 & 1 & -0.066 & 0.018 & 0.004 \\
\hline 5127.90 & C III & 5130.86 & 1 & -0.067 & 0.079 & 0.004 \\
\hline 5137.51 & $\mathrm{C}_{\text {II }}$ & 5140.79 & 1 & -0.069 & 0.046 & 0.009 \\
\hline 5194.45 & {$[\mathrm{~N} \mathrm{I}]$} & 5197.900 & 1 & -0.082 & 0.144 & 0.009 \\
\hline \multirow{2}{*}{5195.10} & {$[\mathrm{NI}]$} & 5197.90 & 2 & -0.082 & 0.366 & 0.010 \\
\hline & & & Tot. & & 0.510 & 0.013 \\
\hline 5196.81 & {$[\mathrm{~N} \mathrm{I}]$} & 5200.26 & 1 & -0.083 & 0.117 & 0.008 \\
\hline \multirow[t]{2}{*}{5197.45} & {$[\mathrm{NI}]$} & 5200.26 & 2 & -0.083 & 0.315 & 0.012 \\
\hline & & & Tot. & & 0.432 & 0.015 \\
\hline 5339.16 & C II & 5342.19 & 1 & -0.112 & 0.078 & 0.008 \\
\hline 5339.19 & C II & 5342.43 & 1 & -0.112 & 0.082 & 0.014 \\
\hline 5408.25 & He II & 5411.52 & 1 & -0.126 & 1.119 & 0.024 \\
\hline 5514.37 & [Cl III] & 5517.66 & 1 & -0.145 & 0.062 & 0.007 \\
\hline 5534.55 & [Cl III] & 5537.60 & 1 & -0.149 & 0.062 & 0.007 \\
\hline 5573.87 & [O I] & 5577.34 & 1 & -0.156 & 0.046 & 0.014 \\
\hline \multirow[t]{2}{*}{5574.30} & {$[\mathrm{O} \mathrm{I}]$} & 5577.34 & 2 & -0.156 & 0.075 & 0.011 \\
\hline & & & Tot. & & 0.120 & 0.018 \\
\hline 5751.02 & {$[\mathrm{~N}$ II $]$} & 5754.64 & 1 & -0.185 & 0.588 & 0.031 \\
\hline \multirow{2}{*}{5751.37} & {$[\mathrm{~N}$ II $]$} & 5754.64 & 2 & -0.185 & 0.903 & 0.030 \\
\hline & & & Tot. & & 1.491 & 0.043 \\
\hline 5797.75 & CIV & 5801.34 & 1 & -0.192 & 0.060 & 0.007 \\
\hline 5843.18 & $\begin{array}{l}{[\mathrm{Xe} I I I]} \\
+\mathrm{He} \text { II }\end{array}$ & 5846.67 & 1 & -0.199 & 0.047 & 0.008 \\
\hline 5872.14 & $\mathrm{He} \mathrm{I}$ & 5875.62 & 1 & -0.203 & 14.918 & 0.363 \\
\hline 6070.48 & He II & 6074.30 & 1 & -0.232 & 0.033 & 0.022 \\
\hline 6147.72 & $\mathrm{C}_{\text {II }}$ & 6151.27 & 1 & -0.242 & 0.059 & 0.009 \\
\hline 6166.94 & He II & 6170.69 & 1 & -0.245 & 0.056 & 0.010 \\
\hline 6230.09 & He II & 6233.82 & 1 & -0.254 & 0.023 & 0.009 \\
\hline 6296.11 & [O I] & 6300.30 & 1 & -0.263 & 1.198 & 0.038 \\
\hline 6296.64 & [O I] & 6300.30 & 2 & -0.263 & 0.520 & 0.113 \\
\hline \multirow[t]{2}{*}{6296.87} & {$[\mathrm{O} \mathrm{I}]$} & 6300.30 & 3 & -0.263 & 4.681 & 0.177 \\
\hline & & & Tot. & & 6.399 & 0.214 \\
\hline 6307.03 & He II & 6310.85 & 1 & -0.264 & 0.050 & 0.010 \\
\hline 6308.17 & [S III $]$ & 6312.10 & 1 & -0.264 & 0.106 & 0.013 \\
\hline 6359.54 & {$[\mathrm{O} \mathrm{I}]$} & 6363.78 & 1 & -0.271 & 0.377 & 0.016 \\
\hline 6359.98 & [O I] & 6363.78 & 2 & -0.271 & 0.134 & 0.041 \\
\hline 6360.30 & [O I] & 6363.78 & 3 & -0.271 & 1.602 & 0.064 \\
\hline & & & Tot. & & 2.114 & 0.077 \\
\hline 6402.47 & He II & 640 & 1 & -0.277 & 0.079 & 0.008 \\
\hline 6457.98 & N II & 6461.71 & 1 & -0.284 & 0.134 & 0.011 \\
\hline 6523.13 & He II & 6527.10 & 1 & -0.293 & 0.091 & 0.011 \\
\hline 6543.91 & {$[\mathrm{~N} \mathrm{II}]$} & 6548.04 & 1 & -0.296 & 8.636 & 0.348 \\
\hline 6544.38 & {$[\mathrm{~N}$ II $]$} & 6548.04 & 2 & -0.296 & 19.071 & 0.623 \\
\hline & & & Tot. & & 27.707 & 0.714 \\
\hline 6556.05 & He II & 6560.10 & 1 & -0.297 & 0.710 & 0.083 \\
\hline 6556.16 & He II & 6560.10 & 2 & -0.297 & 1.142 & 0.068 \\
\hline & & & Tot. & & 1.852 & 0.107 \\
\hline
\end{tabular}

TABLE 1

CONTINUED.

\begin{tabular}{|c|c|c|c|c|c|c|}
\hline $\begin{array}{c}\lambda_{\text {obs }} \\
(\AA)\end{array}$ & Ion & $\begin{array}{l}\lambda_{\text {lab }} \\
(\AA)\end{array}$ & Comp. & $f(\lambda)$ & $I(\lambda)$ & $\delta I(\lambda)$ \\
\hline 6558.82 & $\mathrm{H} 3$ & 6562.77 & 1 & -0.298 & 96.472 & 4.710 \\
\hline 6558.87 & H3 & 6562.77 & $\begin{array}{c}2 \\
\text { Tot. }\end{array}$ & -0.298 & $\begin{array}{l}188.528 \\
285.000\end{array}$ & $\begin{array}{l}6.350 \\
7.906\end{array}$ \\
\hline 6574.09 & C II & 6578.05 & 1 & -0.300 & 0.376 & 0.015 \\
\hline 6579.25 & {$[\mathrm{~N}$ II $]$} & 6583.46 & 1 & -0.300 & 25.484 & 0.965 \\
\hline 6579.73 & {$[\mathrm{~N}$ II $]$} & 6583.46 & $\begin{array}{c}2 \\
\text { Tot. }\end{array}$ & -0.300 & $\begin{array}{l}55.214 \\
80.697\end{array}$ & $\begin{array}{l}1.775 \\
2.021\end{array}$ \\
\hline 6674.13 & $\mathrm{He} \mathrm{I}$ & 6678.15 & 1 & -0.313 & 1.805 & 0.070 \\
\hline 6674.18 & $\mathrm{He} \mathrm{I}$ & 6678.15 & $\begin{array}{c}2 \\
\text { Tot. }\end{array}$ & -0.313 & $\begin{array}{l}1.596 \\
3.401\end{array}$ & $\begin{array}{l}0.056 \\
0.090\end{array}$ \\
\hline 6712.27 & [S II] & 6716.44 & 1 & -0.318 & 0.270 & 0.021 \\
\hline 6712.70 & [S II] & 6716.44 & $\begin{array}{c}2 \\
\text { Tot. }\end{array}$ & -0.318 & $\begin{array}{l}0.235 \\
0.505\end{array}$ & $\begin{array}{l}0.018 \\
0.028\end{array}$ \\
\hline 6726.50 & [S II] & 6730.81 & 1 & -0.320 & 0.170 & 0.014 \\
\hline 6727.02 & [S II] & 6730.81 & $\begin{array}{c}2 \\
\text { Tot. }\end{array}$ & -0.320 & $\begin{array}{l}0.408 \\
0.577\end{array}$ & $\begin{array}{l}0.018 \\
0.023\end{array}$ \\
\hline 6886.73 & He II & 6890.90 & 1 & -0.341 & 0.132 & 0.008 \\
\hline 7061.04 & $\mathrm{He} \mathrm{I}$ & 7065.18 & 1 & -0.364 & 4.345 & 0.160 \\
\hline 7071.72 & {$[\mathrm{Xe} \mathrm{V}] ?$} & 7076.80 & 1 & -0.366 & 0.020 & 0.007 \\
\hline 7104.53 & {$[\mathrm{~K}$ IV $]$} & 7108.90 & 1 & -0.370 & 0.015 & 0.009 \\
\hline 7111.35 & C II & 7115.60 & 1 & -0.371 & 0.020 & 0.007 \\
\hline 7131.50 & [Ar III] & 7135.80 & 1 & -0.374 & 0.348 & 0.016 \\
\hline 7156.36 & $\mathrm{He} \mathrm{I}$ & 7160.61 & 1 & -0.377 & 0.050 & 0.012 \\
\hline 7173.17 & He II & 7177.52 & 1 & -0.379 & 0.112 & 0.007 \\
\hline 7226.94 & C II & 7231.34 & 1 & -0.387 & 0.154 & 0.015 \\
\hline 7232.09 & C II & 7236.42 & 1 & -0.387 & 0.345 & 0.017 \\
\hline 7277.06 & $\mathrm{He} \mathrm{I}$ & 7281.35 & 1 & -0.393 & 0.535 & 0.062 \\
\hline 7285.33 & {$[\mathrm{Rb} \mathrm{V}] ?$} & 7289.81 & 1 & -0.394 & 0.021 & 0.005 \\
\hline 7293.77 & $\mathrm{He} \mathrm{I}$ & 7298.04 & 1 & -0.395 & 0.020 & 0.006 \\
\hline 7314.87 & {$[\mathrm{O} \mathrm{II}]$} & 7319.25 & 1 & -0.398 & 0.933 & 0.058 \\
\hline 7315.51 & [O II] & 7319.89 & 1 & -0.398 & 0.772 & 0.085 \\
\hline \multirow[t]{2}{*}{7315.99} & [O II $]$ & 7320.37 & 2 & -0.398 & 2.193 & 0.108 \\
\hline & & & Tot. & & 2.965 & 0.137 \\
\hline 7325.26 & [O II] & 7329.65 & 1 & -0.400 & 0.753 & 0.064 \\
\hline \multirow[t]{2}{*}{7325.55} & [O II] & 7329.94 & 2 & -0.400 & 0.819 & 0.050 \\
\hline & & & Tot. & & 1.572 & 0.082 \\
\hline 7326.40 & [O II] & 7330.79 & 1 & -0.400 & 0.703 & 0.053 \\
\hline \multirow[t]{2}{*}{7326.62} & [O II $]$ & 7331.01 & 2 & -0.400 & 0.648 & 0.038 \\
\hline & & & Tot. & & 1.351 & 0.065 \\
\hline 7342.94 & {$\left[\begin{array}{ll}\mathrm{V} & \mathrm{II}\end{array}\right]$} & 7347.74 & 1 & -0.402 & 0.057 & 0.010 \\
\hline 7361.03 & O III & 7365.35 & 1 & -0.404 & 0.041 & 0.009 \\
\hline 7369.17 & [V II] & 7373.32 & 1 & -0.406 & 0.056 & 0.008 \\
\hline 7373.23 & [Ni II] & 7377.83 & 1 & -0.406 & 0.026 & 0.011 \\
\hline
\end{tabular}

of narrow Th-Ar comparisons and night sky lines. We took a series of $1800 \mathrm{~s}$ exposures and the total exposure time was $12600 \mathrm{~s}$ (7 exposure frames). In addition, we took a series of four $300 \mathrm{~s}$ exposures to measure the fluxes of strong lines such as $[\mathrm{O} \mathrm{III}] \lambda 5007$. For the flux calibration, blaze function correction, and airmass correction, we observed the standard star Hz44 three times at different airmasses.

The blue spectra (3600-5400 ̊) were obtained in July 2009 using the same settings employed in the May 2009 observations, except for the slit width (1.'2). The seeing during these observations was $0.62^{\prime \prime}$. We took four $1800 \mathrm{~s}$ exposure frames on 4 July and a single $600 \mathrm{~s}$ frame on 9 July.

Data reduction and emission line analysis were performed mainly with the long-slit reduction package noao.twodspec in IRAF 3 . The resulting signal-to-noise $(\mathrm{S} / \mathrm{N})$ ratios at the peak of the detected emission lines in the spectra were $>5$ after subtracting the sky background, whereas that at the continuum was $>40$ at the blue spectrum and $>50$ at the red spectrum before subtracting the sky, even at the edges of each

3 IRAF is distributed by the National Optical Astronomy Observatories, operated by the Association of Universities for Research in Astronomy (AURA), Inc., under a cooperative agreement with the National Science Foundation. 
echelle order.

The line were de-reddened using the formula: $I(\lambda)=$ $F(\lambda) \times 10^{c(\mathrm{H} \beta) f(\lambda)}$, where $I(\lambda)$ is the de-reddened line flux, $F(\lambda)$ is the observed line flux, $f(\lambda)$ is the interstellar extinction parameter at $\lambda$ computed by the reddening law of Cardelli et al. (1989) with $R_{V}=3.1$, and $c(\mathrm{H} \beta)$ is the reddening coefficient at $\mathrm{H} \beta$, respectively. The values of $F(\mathrm{H} \beta)$ were $1.85 \times 10^{-13} \pm 8.49 \times 10^{-15}$ in blue and $2.13 \times 10^{-13} \pm$ $4.60 \times 10^{-15} \mathrm{erg} \mathrm{s}^{-1} \mathrm{~cm}^{-2}$ in red. Hereafter, $\mathrm{X}(-\mathrm{Y})$ means $\mathrm{X} \times 10^{-\mathrm{Y}}$. We measured $c(\mathrm{H} \beta)$ by comparing the observed Balmer line ratios of $\mathrm{H} \gamma$ (blue spectrum) or $\mathrm{H} \alpha$ (red spectrum) to $\mathrm{H} \beta$ with the theoretical ratio of Storey \& Hummer (1995), assuming the electron temperature $T_{\epsilon}=10^{4} \mathrm{~K}$ and the electron density $n_{\epsilon}=10^{4} \mathrm{~cm}^{-3}$ in the Case B assumption. The value of $c(\mathrm{H} \beta)$ was $0.07 \pm 0.05$ for the blue spectra and $0.11 \pm 0.04$ for the red spectra.

Flux scaling was performed using all of the emission lines detected in the overlap region between the blue and the red spectra. The de-reddened fluxes relative to $I(\mathrm{H} \beta)=100$ in both spectra are coincident within $11 \%$ of each other. The combined de-reddened spectrum is presented in Fig. 11 The observed wavelength at the time of observation was corrected to the averaged line-of-sight heliocentric radial velocity of $181.35 \pm 0.35 \mathrm{~km} \mathrm{~s}^{-1}$ (root-mean-square of the residuals: 4.00 $\mathrm{km} \mathrm{s}^{-1}$ ) among over 120 lines detected in the HDS spectrum.

The detected lines are listed in Table 1 To ensure accuracy in the identification, we checked the presence of all of the detected lines and removed ghost emissions in the twodimensional spectra. When measuring fluxes of the emission lines, we assumed that the line profiles were all Gaussian and we applied multiple Gaussian fitting techniques. We listed the observed wavelength and de-reddened relative fluxes of each Gaussian component (indicated by Comp.ID number in the fourth column of Table 1 with respect to the de-reddened $\mathrm{H} \beta$ flux of 100. The line-profiles of most the detected lines can be fit by a single Gaussian component. For the lines composed of multiple components (e.g., [O II] $] 3726.03 \AA$ ), we list the dereddened relative fluxes of each component and list the sum of these components (indicated by Tot.) in the last line.

\subsection{The total $H \beta$ flux}

To normalize the line fluxes relative to $F(\mathrm{H} \beta)$ in the GALEX and Spitzer/IRS spectra, the $F(\mathrm{H} \beta)$ of the whole nebula is needed.

A measurement of $F(\mathrm{H} \beta)=3.16(-13) \mathrm{erg} \mathrm{s}^{-1} \mathrm{~cm}^{-2}$ was taken for the entire nebula by Kwitter et al. (2003) using a slit width of $5^{\prime \prime}$. They also determined $c(\mathrm{H} \beta)=0.10$.

The SDSS spectrum for H4-1 (SDSS; Object ID: 631018077386964992) provides another measurement, taken using a $3^{\prime \prime}$ diameter optical fiber. A total $\mathrm{H} \beta$ flux of 4.57 ($13) \pm 3.13(-15) \mathrm{erg} \mathrm{s}^{-1} \mathrm{~cm}^{-2}$ was measured by applying an aperture correction factor of 2.07, which is listed in the SDSS webpage and is from the $r$-band flux ratio between the image and the fiber spectra. A value of $c(\mathrm{H} \beta)=0.10 \pm 0.04$ was derived by comparing the observed $F(\mathrm{H} \beta) / F(\mathrm{H} \gamma)$ ratio to the theoretical value for the case of $T_{\epsilon}=10^{4} \mathrm{~K}$ and $n_{\epsilon}=10^{4} \mathrm{~cm}^{-3}$, because the $\mathrm{H} \alpha$ line was saturated.

We used a $\mathrm{H} \beta$ flux value of $3.86(-13)$ erg $\mathrm{s}^{-1} \mathrm{~cm}^{-2}$, which is the average between the values from the SDSS and Kwitter et al. (2003).

\subsection{GALEX archive data}

TABLE 2

THE DETECTED LINES IN THE GALEX SPECTRA.

\begin{tabular}{lcccc}
\hline \hline $\begin{array}{c}\lambda_{\text {lab }} \\
(\AA)\end{array}$ & Ion & $f(\lambda)$ & $\begin{array}{c}F(\lambda)^{a} \\
\left(\mathrm{erg} \mathrm{s}^{-1} \mathrm{~cm}^{-2}\right)\end{array}$ & $\begin{array}{c}I(\lambda) \\
{[I(\mathrm{H} \beta)=100]}\end{array}$ \\
\hline $1549 / 51$ & C IV & 1.238 & $1.32(-12) \pm 5.36(-14)$ & $3.42(+2) \pm 1.89(+1)$ \\
1640 & He II & 1.177 & $4.87(-13) \pm 4.38(-14)$ & $1.26(+2) \pm 1.23(+1)$ \\
$1660 / 66$ & [O III $]$ & 1.167 & $2.10(-13) \pm 7.83(-14)$ & $5.43(+1) \pm 2.04(+1)$ \\
$1906 / 09$ & C III] & 1.257 & $6.04(-12) \pm 7.73(-14)$ & $1.56(+3) \pm 6.18(+1)$ \\
2326 & [C II] & 1.364 & $1.68(-12) \pm 1.86(-14)$ & $4.35(+2) \pm 1.70(+1)$ \\
2470 & [O II] & 1.045 & $1.94(-14) \pm 7.00(-15)$ & $5.10(0) \pm 1.82(0)$ \\
\hline
\end{tabular}

a We adopted the $F(\mathrm{H} \beta)=3.86(-13) \mathrm{erg} \mathrm{s}^{-1} \mathrm{~cm}^{-2}$.

TABLE 3

THE DETECTED ATOMIC LINES IN THE Spitzer SPECTRA.

\begin{tabular}{ccccr}
\hline \hline $\begin{array}{c}\lambda_{\text {lab }} \\
(\mu \mathrm{m})\end{array}$ & Ion & $f(\lambda)$ & $\begin{array}{c}F(\lambda)^{a} \\
\left(\mathrm{erg} \mathrm{s}^{-1} \mathrm{~cm}^{-2}\right)\end{array}$ & \multicolumn{1}{c}{$\begin{array}{c}I(\lambda) \\
{[I(\mathrm{H} \beta)=100]}\end{array}$} \\
\hline 10.51 & {$[\mathrm{~S} \mathrm{IV}]$} & -0.960 & $4.97(-15) \pm 4.06(-16)$ & $1.21 \pm 0.15$ \\
12.40 & H I & -0.980 & $4.33(-15) \pm 3.94(-16)$ & $1.04 \pm 0.13$ \\
12.81 & {$[\mathrm{Ne}$ II $]$} & -0.983 & $1.62(-15) \pm 1.85(-16)$ & $0.39 \pm 0.06$ \\
15.56 & {$[\mathrm{Ne}$ III $]$} & -0.985 & $1.48(-14) \pm 3.89(-16)$ & $3.56 \pm 0.34$ \\
16.12 & He I & -0.984 & $3.08(-15) \pm 2.36(-16)$ & $0.74 \pm 0.09$ \\
17.63 & He I & -0.981 & $6.12(-15) \pm 6.05(-16)$ & $1.47 \pm 0.20$ \\
18.71 & {$[\mathrm{~S} \mathrm{III}]$} & -0.981 & $3.80(-15) \pm 2.34(-16)$ & $0.91 \pm 0.10$ \\
25.89 & {$[\mathrm{O}$ IV $]$} & -0.989 & $1.29(-13) \pm 8.57(-16)$ & $30.87 \pm 2.84$ \\
\hline
\end{tabular}

a We adopted the $F(\mathrm{H} \beta)=3.86(-13) \mathrm{erg} \mathrm{s}^{-1} \mathrm{~cm}^{-2}$.

We analyzed the Galaxy Evolution Explorer (GALEX; Martin et al. 2005) slit-less prism spectra to estimate the $\mathrm{CEL} \mathrm{C}^{+, 2+, 3+}$ and $\mathrm{O}^{+}$abundances using the [C II] $\lambda 2326 \AA$, C III] $\lambda 1906 / 09 \AA, \mathrm{CIV} \lambda 1549 / 51 \AA$, and [O II] $\lambda 2470 \AA$ lines. The GALEX spectra were retrieved from the multimission archive at the STScI (MAST; GALEX Object ID: 2555724756316858403). We used both FUV (1344-1786 ^) and NUV (1771-2831 ̊) band spectra. We did not correct the reddening because the observed flux ratio of He II $F(1640$ $\AA) / F(4686 \AA)=6.88 \pm 0.62$ was comparable to the theoretical ratio of He II $I(\lambda 1640) /(\lambda 4686)=6.56$ in the case of $T_{\epsilon}=10^{4} \mathrm{~K}$ and $n_{\epsilon}=10^{4} \mathrm{~cm}^{-3}$ as given by Storey \& Hummer (1995).

The observed and normalized fluxes of the detected lines are listed in Table 2 .

\subsection{Spitzer/IRS archive data}

The spectra were taken on June 12, 2007 using the Infrared Spectrograph (IRS; Houck et al. 2004) with the SL (5.2-14.5 $\mu \mathrm{m})$, SH $(9.9-19.6 \mu \mathrm{m})$, and LH (18.7-37.2 $\mu \mathrm{m})$ modules (AOR Keys: 18628864 and 186291202 ; PI: J. Bernard-Salas). We used the reduction package SMART v.8.2.5 provided by the IRS team at Cornell University (Higdon et al. 2004) and IRSCLEAN provided by the Spitzer Science Center. For SH and LH spectra, we subtracted the sky background using offset spectra. We scaled the SL data up to SH\&LH in the overlapping wavelength region, and then re-scaled the scaled 5.2$37.2 \mu \mathrm{m}$ to match the Wide-field Infrared Survey Explorer (WISE) bands $3\left(\lambda_{c}=11.56 \mu \mathrm{m}\right)$ and $4\left(\lambda_{c}=22.09 \mu \mathrm{m}\right)$ average flux densities $\left(1.91(-13) \mathrm{erg} \mathrm{s}^{-1} \mathrm{~cm}^{-2} \mu \mathrm{m}^{-1}\right.$ in band 3 and $3.16(-13) \mathrm{erg} \mathrm{s}^{-1} \mathrm{~cm}^{-2} \mu \mathrm{m}^{-1}$ in band 4).

The line fluxes of detected atomic lines are listed in Table 3. We derived $c(\mathrm{H} \beta)=0.03 \pm 0.04$ by comparing the observed intensity ratio of $\mathrm{H}$ I $F(12.4 \mu \mathrm{m}) / F(\mathrm{H} \beta)$ to the theoretical value of Storey \& Hummer 1995) for the case of $T_{\epsilon}=10^{4} \mathrm{~K}$ and $n_{\epsilon}=10^{4} \mathrm{~cm}^{-3}$ (1.04). The line at $12.4 \mu \mathrm{m}$ is the complex 


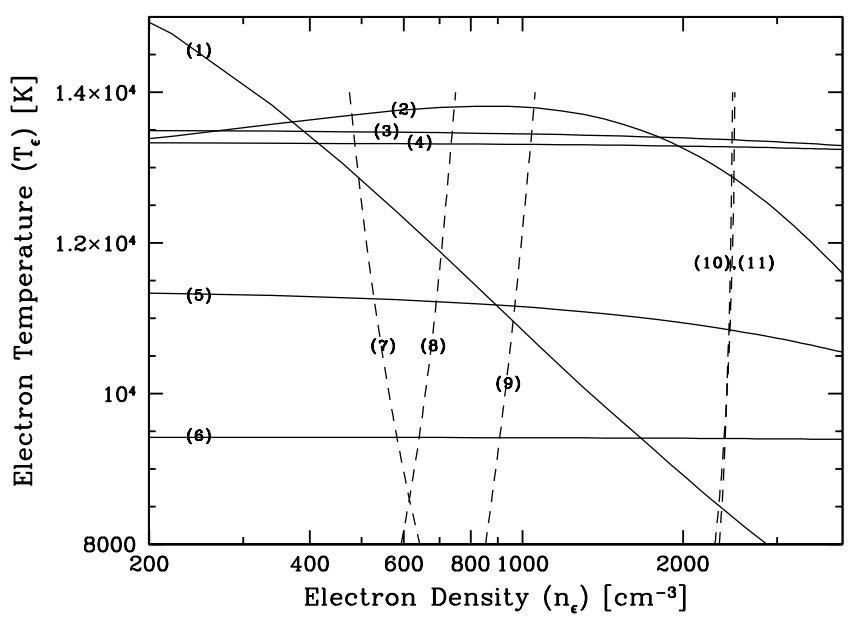

FIG. 2. $-n_{\epsilon}-T_{\epsilon}$ diagram. Each curve is labeled with an ID number given in Table 4 The solid lines indicate diagnostic lines of the $T_{\epsilon}$. The broken lines indicate diagnostic lines of the $n_{\epsilon}$.

of H I $n=9-7$, and $n=11-8$. We used the interstellar extinction function given by Fluks et al. (1994).

\section{RESULTS}

\subsection{CELs Diagnostics}

Electron temperatures and densities were derived from a variety of line diagnostic ratios by solving for level populations using a multi-level ( $>5$ levels) atomic model. The observed diagnostic line ratios are listed in Table 4. The numbers in the second column indicate the ID of each curve in the $n_{\epsilon}-T_{\epsilon}$ diagram presented in Fig. 22 The solid lines indicate diagnostic lines for the electron temperatures, while the broken lines are electron density diagnostics. The third, fourth, and last columns in Table 4 give the diagnostic lines, their line ratios, and the resulting $n_{\epsilon}$ and $T_{\epsilon}$, respectively. The value of $T_{\epsilon}([\mathrm{S}$ III] $)$ was estimated using the observed [S III] $\lambda 18.7 \mu \mathrm{m}$ and $[\mathrm{S} \mathrm{III}] \lambda 9531 \AA$ lines. We adopted $I([\mathrm{~S} \mathrm{III}] \lambda 9531)=2.51$ measured by Kwitter et al. (2003).

For the [O II] $\lambda \lambda 7320 / 30$ line, we subtracted recombination contamination from $\mathrm{O}^{2+}$ using the following equation given by Liu et al. (2000):

$$
\frac{I_{R}([\mathrm{OII}] \lambda \lambda 7320 / 30)}{I(\mathrm{H} \beta)}=9.36\left(\frac{T_{\epsilon}}{10^{4}}\right)^{0.44} \times \frac{\mathrm{O}^{2+}}{\mathrm{H}^{+}} .
$$

Using $\mathrm{O}^{2+}$ ionic abundances derived from the $\mathrm{O}$ II lines and $T_{\epsilon}=10000 \mathrm{~K}$, we estimated that $I_{R}([\mathrm{O}$ II $] \lambda \lambda 7320 / 30)=0.16$, which is a negligible recombination contamination. Because we could not detect the N II and pure O III recombination lines, we did not estimate the contribution of $\mathrm{N}^{2+}$ to the $[\mathrm{N}$ II $] \lambda 5744$ line and of $\mathrm{O}^{3+}$ to the $[\mathrm{O}$ III $] \lambda 4363$ line, respectively.

First, we estimated $n_{\epsilon}$ in $T_{\epsilon}=12000 \mathrm{~K}$ for all density diagnostic lines. The values of $T_{\epsilon}([\mathrm{O} \mathrm{III}]), T_{\epsilon}([\mathrm{Ne} \mathrm{III}])$, and $T_{\epsilon}$ ([S III $\left.]\right)$ were calculated using $n_{\epsilon}=2790 \mathrm{~cm}^{-3}$, which is the averaged $n_{\epsilon}$ between $n_{\epsilon}([\mathrm{Ar} I \mathrm{~V}])$ and $n_{\epsilon}([\mathrm{Cl} \mathrm{III}])$. We calculated $T_{\epsilon}([\mathrm{N} \mathrm{III}])$ and $T_{\epsilon}([\mathrm{O} \mathrm{II}])$ using $n_{\epsilon}([\mathrm{OII}])$, and $T_{\epsilon}([\mathrm{OI}])$ using $n_{\epsilon}([\mathrm{NI}])$.

Our estimated $T_{\epsilon}$ and $n_{\epsilon}$ are comparable to those provided by Kwitter et al. (2003), who estimated $T_{\epsilon}([\mathrm{O}$ III $])=12300 \mathrm{~K}$ and $T_{\epsilon}([\mathrm{N} \mathrm{II}])=10200 \mathrm{~K}$. However, their $T_{\epsilon}([\mathrm{O} \mathrm{II}])(6100 \mathrm{~K})$ was much lower than our calculation, and they also only gave

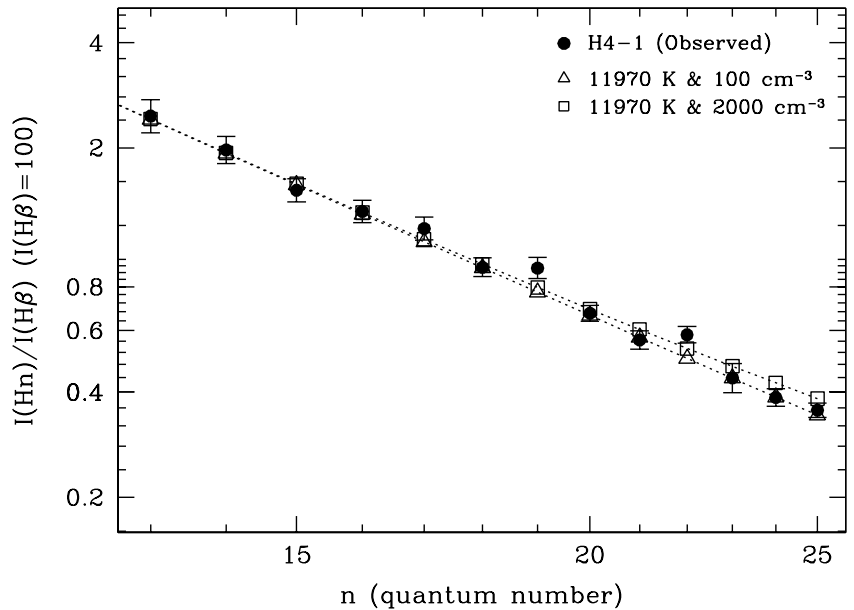

FIG. 3. - Plot of the intensity ratio of the higher order Balmer lines to $\mathrm{H} \beta$ (Case B assumption) with the theoretical intensity ratios in $T_{\epsilon}=11970 \mathrm{~K}$ and $n_{\epsilon}=100$ and $2000 \mathrm{~cm}^{-3}$.

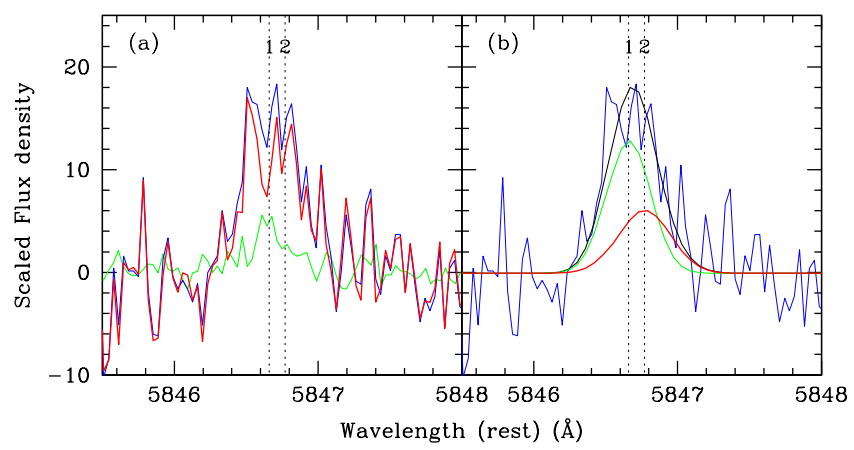

FIG. 4.- (a) The observed line profile at $\lambda 5846.7 \AA$ (blue line). The dotted lines are the rest wavelength of the He II $\lambda 5846.7$ and [Xe III] $\lambda 5846.8$ lines, which are indicated with the numbers 1 and 2, respectively. The line is composed of He II $\lambda 5846.7$ and [Xe III] $\lambda 5846.8$ transitions. The green line is the expected He II $\lambda 5846.7$ profile made by scaling intensity down and shifting wavelength of He II $\lambda 6074.3$ to He II $\lambda 5846$. The red line is the solo [Xe III] $\lambda 5846.8$ (method I). (b) The observed line profile at $\lambda 5846.7 \AA$ (blue line). This line could be represented by two Gaussian components; the green line is He II $\lambda 5846.7$ and the red line is [Xe III] $\lambda 5846.8$. The black line is the sum of these two components (method II, See text in detail).

$n_{\epsilon}([\mathrm{S} \mathrm{II}])\left(400 \mathrm{~cm}^{-3}\right)$.

\subsection{RL Diagnostics}

We calculated $T_{\epsilon}$ using the ratio of the Balmer discontinuity to $I(\mathrm{H} 11)$. The method used to estimate the temperature $T_{\epsilon}(\mathrm{BJ})$ is explained in Liu et al. (2001).

In the case of $n_{\epsilon}=100 \mathrm{~cm}^{-3}$, the He I electron temperatures $T_{\epsilon}(\mathrm{He} \mathrm{I})$ were derived from the ratio He I $I(\lambda 7281) / I(\lambda 6678)$ using the emissivity of He I from Benjamin et al. (1999).

The intensity ratio of a high-order Balmer line Hn (where $\mathrm{n}$ is the principal quantum number of the upper level) to a lower-order Balmer line (e.g., $\mathrm{H} \beta$ ) is also sensitive to the electron density. In Fig. 3. we plot the ratios of higherorder Balmer lines to $\mathrm{H} \beta$ with comparisons to theoretical values from Storey \& Hummer (1995) for values of $T_{\epsilon}(\mathrm{BJ})$ and $n_{\epsilon}=100$ and and $2000 \mathrm{~cm}^{-3}$, respectively. The electron density in the RL emitting region is in the above range.

The $T_{\epsilon}$ and $n_{\epsilon}$ derived from the RL diagnostics are also summarized in Table 4

\subsection{CEL Ionic Abundances}


TABLE 4

Plasma Diagnostics.

\begin{tabular}{|c|c|c|c|c|}
\hline Parameters & ID & Diagnostic & Ratio & Result \\
\hline$T_{\epsilon}$ & (1) & {$[\mathrm{O}$ II $](\lambda 3726+\lambda 3729) /(\lambda 7320+\lambda 7330)$} & $26.459 \pm 1.051^{a}$ & $12000 \pm 330$ \\
\hline \multirow{7}{*}{$(\mathrm{K})$} & (2) & {$[\mathrm{S} \mathrm{III}](\lambda 18.7 \mu \mathrm{m}) /(\lambda 9531)$} & $0.364 \pm 0.099$ & $12600 \pm 2200$ \\
\hline & (3) & {$[\mathrm{Ne} \mathrm{III}](\lambda 15.5 \mu \mathrm{m}) /(\lambda 3869+\lambda 3967)$} & $0.518 \pm 0.052$ & $13400 \pm 500$ \\
\hline & (4) & {$[\mathrm{O} \mathrm{III}](\lambda 4959+\lambda 5007) /(\lambda 4363)$} & $91.390 \pm 3.465$ & $13280 \pm 200$ \\
\hline & (5) & {$[\mathrm{N} \mathrm{II}](\lambda 6548+\lambda 6583) /(\lambda 5755)$} & $72.701 \pm 2.541$ & $11300 \pm 180$ \\
\hline & (6) & {$[\mathrm{O} \mathrm{I}](\lambda 6300+\lambda 6363) /(\lambda 5577)$} & $70.704 \pm 10.527$ & $9490 \pm 510$ \\
\hline & & He I $(\lambda 7281) /(\lambda 6678)$ & $0.157 \pm 0.019$ & $7430 \pm 2190$ \\
\hline & & (Balmer Jump)/(H 11) & $0.103 \pm 0.205$ & $11970 \pm 2900$ \\
\hline$\overline{n_{\epsilon}}$ & (7) & {$[\mathrm{N} \mathrm{I}](\lambda 5198) /(\lambda 5200)$} & $1.182 \pm 0.051$ & $590 \pm 80$ \\
\hline \multirow{5}{*}{$\left(\mathrm{cm}^{-3}\right)$} & (8) & {$[\mathrm{O}$ II $](\lambda 3726) /(\lambda 3729)$} & $1.069 \pm 0.051$ & $710 \pm 110$ \\
\hline & (9) & {$[\mathrm{S}$ II $](\lambda 6716) /(\lambda 6731)$} & $0.875 \pm 0.059$ & $1030 \pm 210$ \\
\hline & (10) & {$[$ Ar IV $](\lambda 4711) /(\lambda 4740)$} & $1.097 \pm 0.143$ & $2750 \pm 1460$ \\
\hline & (11) & {$[\mathrm{Cl} \mathrm{III}](\lambda 5517) /(\lambda 5537)$} & $1.001 \pm 0.158$ & $2840 \pm 1250$ \\
\hline & & Balmer decrement & & $100-2000$ \\
\hline
\end{tabular}

${ }^{a}$ Corrected recombination contribution for [O II] $\lambda \lambda 7320 / 30$.

TABLE 5

THE Adopting ELECTRON TEMPERATURES AND DENSITIES

\begin{tabular}{ccl}
\hline \hline$T_{\epsilon}(\mathrm{K})$ & $n_{\epsilon}\left(\mathrm{cm}^{-3}\right)$ & \multicolumn{1}{c}{ Ions } \\
\hline 9400 & 590 & $\mathrm{~N}^{0}, \mathrm{O}^{0}$ \\
11300 & 710 & $\mathrm{C}^{+}, \mathrm{N}^{+}, \mathrm{O}^{+}$ \\
11300 & 1030 & $\mathrm{~S}^{+}$ \\
13280 & 2790 & $\mathrm{C}^{2+}, \mathrm{C}^{3+}, \mathrm{O}^{2+}, \mathrm{O}^{3+}, \mathrm{Ne}^{+}, \mathrm{Ne}^{2+}$ \\
& & $\mathrm{S}^{2+}, \mathrm{S}^{3+}, \mathrm{Cl}^{2+}, \mathrm{Ar}^{2+}, \mathrm{Ar}^{3+}, \mathrm{Xe}^{2+}$ \\
\hline
\end{tabular}

We obtained 18 ionic abundances: $\mathrm{C}^{+, 2+, 3+}, \mathrm{N}^{0,1+}$, $\mathrm{O}^{0,1+, 2+, 3+}, \mathrm{Ne}^{+, 2+}, \mathrm{S}^{+, 2+, 3+}, \mathrm{Ar}^{2+, 3+}, \mathrm{Cl}^{2+}$, and $\mathrm{Xe}^{2+}$. The estimates of $\mathrm{C}^{+, 3+}, \mathrm{O}^{3+}, \mathrm{Cl}^{2+}$, and $\mathrm{Xe}^{2+}$ are reported for the first time. The ionic abundances were calculated by solving the statistical equilibrium equations for more than five levels in adopting $T_{\epsilon}$ and $n_{\epsilon}$. We used electron temperatures and densities for each ion that were determined using CEL plasma diagnostics. The adopted $T_{\epsilon}$ and $n_{\epsilon}$ values for each ion are listed in Table 5

The derived ionic abundances are presented in Table 6 The last column contains the resulting ionic abundances, $\mathrm{X}^{\mathrm{m}+} / \mathrm{H}^{+}$, and their probable errors, which include errors from line intensities, electron temperature, and electron density. In the last line of each ion's line series, we present the adopted ionic abundance and its error. These values are estimated from the weighted mean of the line intensity.

When calculating the $\mathrm{C}^{+}$abundance, we subtracted contamination from [O III] $\lambda 2321 \AA$ to [C II] $\lambda 2326 \AA$ based on the theoretical intensity ratio of [O III] $I(2326 \AA) / I(4363 \AA)$ $=0.236$. To determine the final $\mathrm{S}^{+}$abundance, we excluded the values derived from the trans-aural line $[\mathrm{S} \mathrm{II}] \lambda 4069 \AA$ because this line would be contaminated from C III $\lambda 4068 \AA$. For $\mathrm{O}^{2+}$, we excluded [O III] $\lambda 1660 / 66 \AA$ because of its large uncertainty.

Dinerstein et al. (2003) reported the upper limit intensities of [Ne II] $\lambda 12.8 \mu \mathrm{m}$ and [S IV] $\lambda 10.5 \mu \mathrm{m}$ and estimated $\mathrm{Ne}^{+} / \mathrm{H}^{+}<4.6(-6)$ and $\mathrm{S}^{3+} / \mathrm{H}^{+}<3.8(-7)$ using the mid-IR spectra taken by TEXES/IRTF. The Spitzer observations improved their estimates.

This is the first detection of [Xe III] $\lambda 5846.8 \AA\left({ }^{3} P_{2}-{ }^{1} D_{2}\right)$ in H4-1. The detection of this element is very rare because there are only a handful of detection cases in $\mathrm{PNe}$ (Sharpee et al. 2007; Sterling et al. 2009; García-Rojas et al. 2012; Otsuka et al. 2010).

The local continuum subtracted $[\mathrm{Xe}$ III] $\lambda 5846.8$ line profile is presented in Fig. 4 In high-excitation PNe such as H41, this line appears to be blended with He II $\lambda 5846.7$ ( $n=31-5$; Pfund series). The blue line in Fig. 4(a) indicates the observed line profile. The [Fe II] $\lambda 5847.3$ line possibly contributes to the emission line at $\lambda 5846$, however, this line has poorer wavelength agreement and our photo-ionization model of H4-1 using CLOUDY code (Ferland et al. 1998) predicted that the intensity ratio of $I([\mathrm{Fe} \mathrm{II}] \lambda 5846.7)$ to $I(\mathrm{H} \beta)$ is $<1(-8)$. Therefore, the emission line at $\lambda 5846$ would be a combination of the $[\mathrm{Xe} I I I]$ and He II lines.

We attempted to obtain the solo [Xe III] $\lambda 5846.8$ line flux by subtracting the contribution from He II $\lambda 5846.7$ using the following method (method I). First, we scaled the intensity of He II $\lambda 6074.3$ down to He II $\lambda 5846.7$ using the theoretical ratio of $I(\lambda 5846.7) / I(6074.3)$, which is 0.271 in the case of $T_{\epsilon}=11970 \mathrm{~K}\left(=T_{\epsilon}(\mathrm{BJ})\right)$ and $1000 \mathrm{~cm}^{-3}$ according to Storey \& Hummer (1995). Second, we shifted the intensity peak wavelength of this scaled line to $5846.7 \AA$. The predicted He II $\lambda 5846.7$ is indicated by the green line in Fig. 4 a). Next, we subtracted this shifted line from the $5846 \AA$ line. Finally, we obtained an intensity of the [Xe III] $\lambda 5846.8$ line of 3.63($2) \pm 1.04(-2)$, as indicated by the red line in Fig. 4 (a).

We also attempted to obtain the [Xe III] $\lambda 5846.8$ using Gaussian fitting, as shown in Fig. 4(b) (method II). As constraints, we fix the rest wavelengths of the intensity peak and FWHM velocities to equal $5846.7 \AA$ and $18.33 \mathrm{~km} \mathrm{~s}^{-1}$ for He II $\lambda 5846.7$, respectively, and 5846.8 and $21.90 \mathrm{~km}$ $\mathrm{s}^{-1}$ for [Xe III] $\lambda 5846.8$. Here, $18.33 \mathrm{~km} \mathrm{~s}^{-1}$ and $21.90 \mathrm{~km}$ $\mathrm{s}^{-1}$ correspond to the FWHM velocities of He II $\lambda 6074.3$ and $[\mathrm{Cl}$ III $] \lambda 5537$ (I.P.=23.81 eV). Because the I.P. of $\mathrm{Xe}^{2+}(21.21$ $\mathrm{eV}$ ) is very close to $\mathrm{Cl}^{2+}$, we assumed that the FWHM velocity of $[\mathrm{Xe} \mathrm{III}]$ is almost identical to that of $[\mathrm{Cl} \mathrm{III}] \lambda 5537$. In method II, we obtained $I([\mathrm{Xe} I \mathrm{II}] \lambda 5846.8)>1.42(-2)$.

Based on the above analyses, it is probable that [Xe III] $\lambda 5846.8$ would be in the HDS spectrum. However, we need to carefully consider the estimation of the $[\mathrm{Xe}$ III $] \lambda 5846.8$ line intensity; because we could not detect any Pfund series He II lines with an upper $n$ from 29 to 21 and $>32$, He II $\lambda 5846.7$ may be not in the HDS spectrum or it may be present at a very low intensity. The emission line at $5846.8 \AA$ would be mostly from the [Xe III] $\lambda 5846.8$ line. In this work, we determined the lower limit $\mathrm{Xe}^{2+}$ and $\mathrm{Xe}$ abundances using method I based on the transition probabilities of Biemont et al. (1995) and the collisional impacts of Schoening \& Butler (1998).

We could not retrieve strong lines for Ba II $\lambda 4934.6 \AA$ 
TABLE 6

IONIC ABUNDANCES FROM CELS

\begin{tabular}{|c|c|c|c|}
\hline $\mathrm{X}^{\mathrm{m}+}$ & $\lambda_{\text {lab }}$ & $I\left(\lambda_{\mathrm{lab}}\right)$ & $\mathrm{X}^{\mathrm{m}+} / \mathrm{H}^{+}$ \\
\hline $\mathrm{C}^{+}$ & $2326 \AA$ & $4.35(+2) \pm 1.70(+1)$ & $4.00(-4) \pm 3.88(-5)$ \\
\hline $\mathrm{C}^{2+}$ & 1906/9 A & $1.56(+3) \pm 6.18(+2)$ & $5.51(-4) \pm 5.23(-5)$ \\
\hline $\mathrm{C}^{3+}$ & $1549 / 51 \AA$ & $3.42(+2) \pm 1.89(+1)$ & $8.85(-5) \pm 1.04(-5)$ \\
\hline \multirow[t]{2}{*}{$\mathrm{N}^{0}$} & $5197.9 \AA$ & $5.10(-1) \pm 1.33(-2)$ & $8.88(-7) \pm 1.77(-7)$ \\
\hline & $5200.3 \AA$ & $4.32(-1) \pm 1.47(-2)$ & $\begin{array}{l}8.93(-7) \pm 1.72(-7) \\
\mathbf{8 . 9 1 ( - 7 ) \pm 1 . 7 5 ( - 7 )}\end{array}$ \\
\hline \multirow[t]{3}{*}{$\mathrm{N}^{+}$} & $5754.6 \AA$ & $1.49(0) \pm 4.30(-2)$ & $1.17(-5) \pm 8.85(-7)$ \\
\hline & $6548.0 \AA$ & $2.77(+1) \pm 7.14(-1)$ & $1.19(-5) \pm 5.37(-7)$ \\
\hline & $6583.5 \AA$ & $8.07(+1) \pm 2.02(0)$ & $\begin{array}{l}1.17(-5) \pm 5.25(-7) \\
\mathbf{1 . 1 8}(-\mathbf{5}) \pm \mathbf{5 . 3 3}(-7)\end{array}$ \\
\hline \multirow[t]{3}{*}{$\mathrm{O}^{0}$} & $5577.3 \AA$ & $1.20(-1) \pm 1.76(-2)$ & $1.49(-5) \pm 4.84(-6)$ \\
\hline & $6300.3 \AA$ & $6.40(0) \pm 2.14(-1)$ & $1.51(-5) \pm 2.80(-6)$ \\
\hline & $6363.8 \AA$ & $2.11(0) \pm 7.72(-2)$ & $\begin{array}{l}1.56(-5) \pm 2.91(-6) \\
\mathbf{1 . 5 2 ( - 5 )} \pm \mathbf{2 . 8 6}(-6)\end{array}$ \\
\hline \multirow[t]{4}{*}{$\mathrm{O}^{+}$} & $3726.0 \AA$ & $9.11(+1) \pm 3.33(0)$ & $4.55(-5) \pm 3.14(-6)$ \\
\hline & $3728.8 \AA$ & $8.52(+1) \pm 2.61(0)$ & $4.58(-5) \pm 3.12(-6)$ \\
\hline & $7320 / 30 \AA$ & $6.66(0) \pm 2.11(-1)$ & $4.98(-5) \pm 4.39(-6)$ \\
\hline & $2470 \AA$ & $5.10(0) \pm 1.82(0)$ & $\begin{array}{l}5.07(-5) \pm 1.82(-5) \\
\mathbf{4 . 5 8}(-\mathbf{5}) \pm \mathbf{3 . 1 6}(-6)\end{array}$ \\
\hline \multirow[t]{5}{*}{$\mathrm{O}^{2+}$} & $1660 / 66 \AA$ & $5.43(+1) \pm 2.03(+1)$ & $2.91(-4) \pm 1.12(-4)$ \\
\hline & $4361.2 \AA$ & $9.76(0) \pm 2.31(-1)$ & $9.66(-5) \pm 7.63(-6)$ \\
\hline & $4931.8 \AA$ & $7.33(-2) \pm 1.28(-2)$ & $7.93(-5) \pm 1.42(-5)$ \\
\hline & $4958.9 \AA$ & $2.24(+2) \pm 3.10(0)$ & $9.46(-5) \pm 4.01(-6)$ \\
\hline & $5006.8 \AA$ & $6.67(+2) \pm 2.62(+1)$ & $\begin{array}{l}9.76(-5) \pm 5.50(-6) \\
\mathbf{9 . 6 9 ( - 5 )} \pm \mathbf{5 . 1 5}(-\mathbf{6})\end{array}$ \\
\hline $\mathrm{O}^{3+}$ & $25.9 \mu \mathrm{m}$ & $3.09(+1) \pm 2.84(0)$ & $7.56(-6) \pm 7.01(-7)$ \\
\hline $\mathrm{Ne}^{+}$ & $12.8 \mu \mathrm{m}$ & $3.91(-1) \pm 5.70(-2)$ & $4.50(-7) \pm 6.58(-8)$ \\
\hline \multirow[t]{3}{*}{$\mathrm{Ne}^{2+}$} & $3868.8 \AA$ & $5.32(0) \pm 2.25(-1)$ & $2.26(-6) \pm 1.43(-7)$ \\
\hline & $3967.5 \AA$ & $1.56(0) \pm 5.90(-2)$ & $1.59(-6) \pm 9.58(-8)$ \\
\hline & $15.6 \mu \mathrm{m}$ & $3.56(0) \pm 3.39(-1)$ & $\begin{array}{c}2.03(-6) \pm 1.94(-7) \\
\mathbf{2 . 0 8}(-\mathbf{6}) \pm \mathbf{1 . 5 3}(-7)\end{array}$ \\
\hline \multirow[t]{3}{*}{$\mathrm{S}^{+}$} & $4068.6 \AA$ & $2.77(-1) \pm 1.92(-2)$ & $5.19(-8) \pm 4.44(-9)$ \\
\hline & $6716.4 \AA$ & $5.05(-1) \pm 2.76(-2)$ & $2.23(-8) \pm 1.46(-9)$ \\
\hline & $6730.8 \AA$ & $5.77(-1) \pm 2.31(-2)$ & 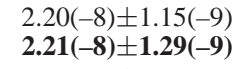 \\
\hline \multirow[t]{2}{*}{$\mathrm{S}^{2+}$} & $6312.1 \AA$ & $1.06(-1) \pm 1.29(-2)$ & $8.15(-8) \pm 1.07(-8)$ \\
\hline & $18.7 \mu \mathrm{m}$ & $9.15(-1) \pm 1.00(-1)$ & $\begin{array}{l}8.17(-8) \pm 9.01(-9) \\
\mathbf{8 . 1 7}(-8) \pm \mathbf{9 . 1 9 ( - 9 )}\end{array}$ \\
\hline $\mathrm{S}^{3+}$ & $10.5 \mu \mathrm{m}$ & $1.77(0) \pm 2.41(-1)$ & $4.77(-8) \pm 6.50(-9)$ \\
\hline \multirow[t]{2}{*}{$\mathrm{Cl}^{2+}$} & $5517.7 \AA$ & $6.16(-2) \pm 7.19(-3)$ & $3.71(-9) \pm 4.52(-10)$ \\
\hline & $5537.6 \AA$ & $6.16(-2) \pm 6.60(-3)$ & $\begin{array}{l}3.60(-9) \pm 4.05(-10) \\
\mathbf{3 . 6 5 ( - 9 )} \pm \mathbf{4 . 2 9 ( - 1 0 )}\end{array}$ \\
\hline $\mathrm{Ar}^{2+}$ & $7135.8 \AA$ & $3.48(-1) \pm 1.56(-2)$ & $1.75(-8) \pm 9.27(-10)$ \\
\hline \multirow[t]{2}{*}{$\mathrm{Ar}^{3+}$} & $4711.4 \AA$ & $6.52(-2) \pm 7.00(-3)$ & $7.72(-9) \pm 8.88(-10)$ \\
\hline & $4740.2 \AA$ & $5.94(-2) \pm 4.35(-3)$ & $\begin{array}{l}7.56(-9) \pm 6.33(-10) \\
7.65(-9) \pm 7.67(-10)\end{array}$ \\
\hline $\mathrm{Xe}^{2+}$ & $5846.7 \AA$ & $>2.59(-2)$ & $>2.95(-10)^{\mathrm{a}}$ \\
\hline $\mathrm{Ba}^{+}$ & $6141.7 \AA$ & $<9.83(-3)$ & $<3.23(-11)^{\mathrm{b}}$ \\
\hline
\end{tabular}

a from method I.

b The value from 1- $\sigma$ flux density around $6141.7 \AA$. See text in detail.

and $6141.7 \AA$ from the HDS spectrum because both lines were deeply affected by moonlight. Therefore, we calculated an upper limit for the expected $\mathrm{Ba}^{+}$abundance of 3.23(10) using the $1-\sigma$ flux density at $\mathrm{Ba}$ II $\lambda 6141.7$ (I)6141.7 $\AA) / I(\mathrm{H} \beta)=9.83(-3), I(\mathrm{H} \beta)=100)$ with $T_{\epsilon}=11300 \mathrm{~K}, n_{\epsilon}=1030$ $\mathrm{cm}^{-3}$, and FWHM velocity $=19 \mathrm{~km} \mathrm{~s}^{-1}$. In the Ba ${ }^{+}$calculation, we used transition probabilities from Klose et al. (2002) and collisional impacts from Schoening \& Butler (1998)

\subsection{RL Ionic Abundances}

The estimated RL ionic abundances are listed in Table 7 The calculations of $\mathrm{C}^{3+, 4+}, \mathrm{N}^{3+}$, and $\mathrm{O}^{2+, 3+}$ abundances were performed for the first time. The Case $\mathrm{B}$ assumption applies to lines from levels that have the same spin as the ground state,
TABLE 7

IONIC ABUNDANCES FROM RLS.

\begin{tabular}{|c|c|c|c|c|}
\hline $\mathrm{X}^{\mathrm{m}+}$ & Multi. & $\lambda_{\text {lab }}$ & $I\left(\lambda_{\text {lab }}\right)$ & $\mathrm{X}^{\mathrm{m}+} / \mathrm{H}^{+}$ \\
\hline \multirow[t]{5}{*}{$\mathrm{He}^{+}$} & V11 & $5875.62 \AA$ & $1.49(+1) \pm 3.63(-1)$ & $1.00(-1) \pm 4.09(-2)$ \\
\hline & V14 & $4471.47 \AA$ & $4.49(0) \pm 9.31(-2)$ & $8.46(-2) \pm 3.20(-2)$ \\
\hline & V46 & $6678.15 \AA$ & $3.40(0) \pm 9.01(-2)$ & $7.97(-2) \pm 3.27(-2)$ \\
\hline & V48 & $4921.93 \AA$ & $1.01(0) \pm 1.70(-2)$ & $7.52(-2) \pm 3.34(-2)$ \\
\hline & V51 & $4387.93 \AA$ & $5.32(-2) \pm 1.64(-2)$ & $\begin{array}{l}8.68(-2) \pm 3.86(-2) \\
9.30(-2) \pm \mathbf{3 . 7 7}(-2)\end{array}$ \\
\hline $\mathrm{He}^{2+}$ & 3.4 & $4685.68 \AA$ & $1.83(+1) \pm 2.27(-1)$ & $1.54(-2) \pm 2.04(-3)$ \\
\hline \multirow[t]{6}{*}{$\mathrm{C}^{2+}$} & V4 & $3920.68 \AA$ & $4.97(-2) \pm 1.84(-2)$ & $1.17(-3) \pm 4.94(-4)$ \\
\hline & V6 & $4267.15 \AA$ & $1.12(0) \pm 3.50(-2)$ & $1.15(-3) \pm 1.80(-4)$ \\
\hline & V16.04 & $6151.27 \AA$ & $5.93(-2) \pm 9.11(-3)$ & $1.36(-3) \pm 2.79(-4)$ \\
\hline & V17.04 & $6461.71 \AA$ & $1.34(-1) \pm 1.07(-2)$ & $1.33(-3) \pm 2.59(-4)$ \\
\hline & V17.06 & $5342.19 \AA$ & $7.85(-2) \pm 7.72(-3)$ & $1.49(-3) \pm 2.99(-4)$ \\
\hline & V17.06 & $5342.43 \AA$ & $8.23(-2) \pm 1.43(-2)$ & $\begin{array}{l}1.56(-3) \pm 3.85(-4) \\
\mathbf{1 . 2 1}(-\mathbf{3}) \pm \mathbf{2 . 1 9}(-\mathbf{4})\end{array}$ \\
\hline \multirow[t]{4}{*}{$\mathrm{C}^{2+}$} & V1 & $4647.42 \AA$ & $2.32(-1) \pm 7.15(-3)$ & $3.64(-4) \pm 4.37(-5)$ \\
\hline & V1 & $4650.25 \AA$ & $1.47(-1) \pm 5.89(-3)$ & $3.86(-4) \pm 4.74(-5)$ \\
\hline & V16 & $4067.87 \AA$ & $1.62(-1) \pm 1.51(-2)$ & $4.25(-4) \pm 7.22(-5)$ \\
\hline & V16 & $4070.14 \AA$ & $2.61(-1) \pm 1.76(-2)$ & $\begin{array}{l}3.82(-4) \pm 6.00(-5) \\
\mathbf{3 . 8 6}(-\mathbf{4}) \pm \mathbf{5 . 5 5}(\mathbf{- 5})\end{array}$ \\
\hline $\mathrm{C}^{3+}$ & V1 & $4658.20 \AA$ & $6.48(-2) \pm 4.19(-3)$ & $1.55(-5) \pm 2.45(-6)$ \\
\hline \multirow[t]{2}{*}{$\mathrm{N}^{3+}$} & $\mathrm{V} 2$ & $4634.12 \AA$ & $8.27(-2) \pm 1.11(-2)$ & $6.51(-5) \pm 1.22(-5)$ \\
\hline & $\mathrm{V} 2$ & $4640.64 \AA$ & $1.48(-1) \pm 5.84(-3)$ & $\begin{array}{l}6.50(-5) \pm 8.88(-6) \\
\mathbf{6 . 5 0}(-\mathbf{5}) \pm \mathbf{1 . 0 1}(-\mathbf{5})\end{array}$ \\
\hline \multirow[t]{5}{*}{$\mathrm{O}^{2+}$} & V1 & $4638.86 \AA$ & $5.71(-2) \pm 5.99(-3)$ & $3.51(-4) \pm 5.74(-5)$ \\
\hline & V1 & $4641.81 \AA$ & $4.72(-2) \pm 4.90(-3)$ & $1.81(-4) \pm 2.32(-5)$ \\
\hline & V1 & $4651.33 \AA$ & $2.36(-2) \pm 5.42(-3)$ & $1.40(-4) \pm 3.66(-5)$ \\
\hline & V1 & $4661.63 \AA$ & $3.53(-2) \pm 5.14(-3)$ & $1.96(-4) \pm 3.77(-5)$ \\
\hline & $\mathrm{V} 2$ & $4349.43 \AA$ & $2.80(-2) \pm 5.97(-3)$ & $\begin{array}{l}1.41(-4) \pm 3.49(-5) \\
\mathbf{1 . 6 9 ( - 4 )} \pm \mathbf{3 . 4 0}(-\mathbf{5})\end{array}$ \\
\hline \multirow[t]{2}{*}{$\mathrm{O}^{3+}$} & $\mathrm{V} 2$ & $3754.70 \AA$ & $1.82(-1) \pm 1.97(-2)$ & $3.47(-4) \pm 5.80(-5)$ \\
\hline & V2 & $3757.24 \AA$ & $7.79(-2) \pm 1.27(-2)$ & $\begin{array}{l}3.35(-4) \pm 6.92(-5) \\
\mathbf{3 . 4 3}(-\mathbf{4}) \pm \mathbf{6 . 1 3}(\mathbf{- 5})\end{array}$ \\
\hline
\end{tabular}

and the Case A assumption applies to lines of other multiplicities. In the last line of each ion's line series, we present the adopted ionic abundance and the error estimate from the line intensity-weighted mean. Because the RL ionic abundances are insensitive to the electron density under $\lesssim 10^{8} \mathrm{~cm}^{-3}$, we adopted $n_{\epsilon}=10^{4} \mathrm{~cm}^{-3}$ for $\mathrm{He}^{2+}, \mathrm{C}^{2+, 3+, 4+}, \mathrm{N}^{3+}$, and $\mathrm{O}^{2+, 3+}$ and $n_{\epsilon}=10^{2} \mathrm{~cm}^{-3}$ for $\mathrm{He}^{+}$. The emission coefficients are the same as those used in Otsuka et al. (2010).

We detected multiplet V2 N III lines, however, these lines are not recombination lines, but resonance lines. Because we detected the $\mathrm{O}$ III resonance line, the intensity of the resonance line N III $\lambda 374.36 \AA\left(2 p^{2} P^{0}-3 d^{2} D\right)$ is enhanced by O III resonance lines at the wavelength of $374.11 \AA\left(2 p^{2}{ }^{3} P\right.$ $\left.3 s^{3} P^{0}\right)$. The line intensities of the multiplet V2 lines can be enhanced by $\mathrm{O}$ III lines. Furthermore, the detected V2 O III lines might be excited by the Bowen fluorescence mechanism or by the charge exchange of $\mathrm{O}^{3+}$ and $\mathrm{H}^{0}$ instead of by recombination. Therefore, we did not use the $\mathrm{N}^{3+}$ and $\mathrm{O}^{3+}$ abundances to determine elemental RL $\mathrm{N}$ and $\mathrm{O}$ abundances.

We detected the multiplet V1 and V2 O II lines. Ruiz et al. (2003), Peimbert et al. (2005), and García-Rojas et al. (2009) pointed out that the upper levels of the transitions in the V1 O II line are not in local thermal equilibrium (LTE) for $n_{\epsilon}<$ $10000 \mathrm{~cm}^{-3}$, and that the abundances derived from each individual line could differ by a factor of $\sim 4$ (García-Rojas et al. 2009). However, the V2 lines are not affected by non-LTE effects. Because H4-1 is a low-density PN $\left(<3000 \mathrm{~cm}^{-3}\right)$, we performed the non-LTE corrections using equations (8) 
TABLE 8

ADOPTED IONIZATION CORRECTION FACTORS (ICFS).

\begin{tabular}{lccl}
\hline \hline $\mathrm{X}$ & Line & $\mathrm{ICF}(\mathrm{X})$ & \multicolumn{1}{c}{$\mathrm{X} / \mathrm{H}$} \\
\hline $\mathrm{He}$ & $\mathrm{RL}$ & $\frac{1}{1-\left(\frac{\mathrm{C}^{4+}}{\mathrm{C}}\right)_{\mathrm{RL}}}$ & $\mathrm{He}+\mathrm{He}^{2+}$ \\
$\mathrm{C}$ & $\mathrm{CEL}$ & $\frac{1}{1-\left(\frac{\mathrm{N}^{+}}{\mathrm{N}}\right)_{\mathrm{CEL}}}$ & $\mathrm{ICF}(\mathrm{C})\left(\mathrm{C}^{2+}+\mathrm{C}^{2+}+\mathrm{C}^{3+}\right)$ \\
& $\mathrm{RL}$ & $\left(\frac{\mathrm{O}}{\mathrm{O}^{+}}\right)_{\mathrm{CEL}}$ & $\mathrm{ICF}(\mathrm{N}) \mathrm{N}^{+}$ \\
$\mathrm{N}$ & $\mathrm{CEL}$ & $\left(\frac{\mathrm{O}}{\mathrm{O}^{2+}}\right)_{\mathrm{CEL}}$ & $\mathrm{O}+\mathrm{O}^{2+}+\mathrm{O}^{3+}$ \\
$\mathrm{O}$ & $\mathrm{CEL}$ & $\left(\frac{\mathrm{O}}{\mathrm{O}^{+}+\mathrm{O}^{2+}}\right)_{\mathrm{CEL}}$ & $\mathrm{ICF}(\mathrm{Ne})\left(\mathrm{Ne}^{+}+\mathrm{Ne}^{2+}\right)$ \\
& $\mathrm{RL}$ & $\left(\frac{\mathrm{Ar}}{\mathrm{Ar}{ }^{2+}}\right)$ & $\mathrm{S}^{+}+\mathrm{S}^{2+}+\mathrm{S}^{3+}$ \\
$\mathrm{Ne}$ & $\mathrm{CEL}$ & $\mathrm{ICF}(\mathrm{Cl}) \mathrm{Cl}^{2+}$ \\
$\mathrm{S}$ & $\mathrm{CEL}$ & $\frac{1}{1-\left(\frac{\mathrm{N}^{+}}{\mathrm{N}}\right)_{\mathrm{CEL}}}$ & $\mathrm{ICF}(\mathrm{Ar})\left(\mathrm{Ar}^{2+}+\mathrm{Ar}^{3+}\right)$ \\
$\mathrm{Cl}$ & $\mathrm{CEL}$ & $\left(\frac{\mathrm{Ar}}{\mathrm{Ar}}\right)$ & $\mathrm{ICF}(\mathrm{Xe}) \mathrm{Xe}^{2+}$ \\
$\mathrm{Ar}$ & $\mathrm{CEL}$ & 1 & $\mathrm{ICF}(\mathrm{Ba}) \mathrm{Ba}^{+}$ \\
$\mathrm{Xe}$ & $\mathrm{CEL}$ & & \\
$\mathrm{Ba}$ & $\mathrm{CEL}$ &
\end{tabular}

through (10) from Peimbert et al. (2005) with $n_{\epsilon}=2000 \mathrm{~cm}^{-3}$. The resulting $\mathrm{O}^{2+}$ abundances determined using the $\mathrm{V} 1$ and V2 lines are in good agreement, except for the O II $\lambda 4638.86$ line. This line was therefore excluded in the final determination of the $\mathrm{RL} \mathrm{O}^{2+}$ abundance.

\subsection{Elemental Abundances}

The elemental abundances were estimated using an ionization correction factor, $\operatorname{ICF}(\mathrm{X})$, which is based on the ionization potential. The $\mathrm{ICF}(\mathrm{X})$ for each element is listed in Table 8

The He abundance is the sum of the $\mathrm{He}^{+}$and $\mathrm{He}^{2+}$ abundances. We assume that the $\mathrm{C}$ abundance is the sum of the $\mathrm{C}^{+}, \mathrm{C}^{2+}, \mathrm{C}^{3+}$, and $\mathrm{C}^{4+}$ abundances, and we corrected for the CEL $\mathrm{C}^{4+}$ and the $\mathrm{RL} \mathrm{C}^{+}$. The $\mathrm{N}$ abundance is the sum of $\mathrm{N}^{+}$, $\mathrm{N}^{2+}$, and $\mathrm{N}^{3+}$, and we corrected for the $\mathrm{N}^{2+}$ and $\mathrm{N}^{3+}$ abundances. The $\mathrm{O}$ abundance is the sum of the $\mathrm{O}^{+}, \mathrm{O}^{2+}$, and $\mathrm{O}^{3+}$ abundances. For the RL $\mathrm{O}$ abundance, we estimated the $\mathrm{O}^{+}$ and $\mathrm{O}^{3+}$ abundances. The $\mathrm{Ne}$ abundance is the sum of the $\mathrm{Ne}^{+}, \mathrm{Ne}^{2+}$, and $\mathrm{Ne}^{3+}$ abundances, and we corrected for the $\mathrm{Ne}^{3+}$ abundance. The $\mathrm{S}$ abundance is the sum of the $\mathrm{S}^{+}, \mathrm{S}^{2+}$, and $\mathrm{S}^{3+}$ abundances. The $\mathrm{Cl}$ abundance is the sum of the $\mathrm{Cl}^{+}$, $\mathrm{Cl}^{2+}, \mathrm{Cl}^{3+}$, and $\mathrm{Cl}^{4+}$ abundances, correcting for $\mathrm{Cl}^{+}, \mathrm{Cl}^{3+}$, and $\mathrm{Cl}^{3+}$. The $\mathrm{Ar}$ abundance is the sum of the $\mathrm{Ar}^{+}, \mathrm{Ar}^{2+}, \mathrm{Ar}^{3+}$, and $\mathrm{Ar}^{4+}$ abundances, correcting for the $\mathrm{Ar}^{+}$and $\mathrm{Ar}^{4+}$ abundances. The Xe abundance is the sum of the $\mathrm{Xe}^{+}, \mathrm{Xe}^{2+}, \mathrm{Xe}^{3+}$, and $\mathrm{Xe}^{4+}$ abundances, correcting for the $\mathrm{Xe}^{+}, \mathrm{Xe}^{3+}$, and $\mathrm{Xe}^{4+}$ abundances. We did not correct for the ionization of $\mathrm{Ba}$.

The resulting elemental abundances are listed in Table 9 The types of emission lines used for the abundance estimations are specified in the second column. The number densities of each element relative to hydrogen are listed in the third column. The fourth column lists the number densities and the fifth column lists the number densities relative to the solar value. The last two columns are the solar abundances and the adopted ICF values. We referred to Asplund et al. (2009) for $\mathrm{N}$ and $\mathrm{Cl}$, and Lodders (2003) for the other elements.

The $[\mathrm{C} / \mathrm{O}]$ abundances are $+1.25 \pm 0.19$ dex from the $\mathrm{RL}$ and $+1.14 \pm 0.20$ dex from the CEL, therefore H4- 1 is an extremely C-enhanced PN. However, as shown in the fifth column of Table 9 , the $\mathrm{C}$ and $\mathrm{O}$ abundances are slightly different between RLs and CELs. The $\mathrm{C}$ and $\mathrm{O}$ abundance discrepan- cies could be explained by small temperature fluctuations in the nebula. We discuss the $\mathrm{C}$ and $\mathrm{O}$ abundance discrepancies in the next section.

In Table [10, we compiled results for H4-1. We improved the $\mathrm{C}, \mathrm{O}, \mathrm{Ne}, \mathrm{S}, \mathrm{Ar}$ abundances and newly added the $\mathrm{Cl}$ and $\mathrm{Xe}$ abundances, thanks to high-dispersion Subaru/HDS spectra and the detection of many different ionization stage ions. The large discrepancy in $\mathrm{C}$ abundance between the RL (Torres-Peimbert \& Peimbert 1979) and the CEL (Henry et al. 1996) is reduced as a result of our work.

In Table 11, we summarize the Xe abundances in 11 Galactic PNe. Using the line lists from García-Rojas et al. (2012), we estimated the Xe abundances of M3-15, NGC5189, $\mathrm{PC} 14$, and $\mathrm{Pe} 1-1$ in $T_{\epsilon}([\mathrm{OIII}])$ and $n_{\epsilon}([\mathrm{Cl} \mathrm{III}])$. For the above PNe, we removed the contribution of He II $\lambda 5846.7$ to [Xe III] $\lambda 5846.8$ using method I as described in Section 3.3. The Xe abundance in H4-1 is the highest among these PNe. In the next section, we also discuss how much Xe in H4-1 is a product of AGB nucleosynthesis in the progenitor.

\section{DISCUSSION}

\section{1. $C$ and $O$ abundance discrepancies}

The RL to CEL abundance ratio, also known as the abundance discrepancy factor $(\mathrm{ADF})$, in $\mathrm{C}^{2+}, \mathrm{C}^{3+}$, and $\mathrm{O}^{2+}$ is $2.20 \pm 0.45,4.36 \pm 0.81$, and $1.75 \pm 0.36$, respectively. For most PNe, ADFs are typically between 1.6 and 3.2 (see Liu 2006).

The value of $T_{\epsilon}(\mathrm{He} \mathrm{I})$ is comparable to the value of $T_{\epsilon}(\mathrm{BJ})$ within estimation error. According to Zhang et al. (2005a), if $T_{\epsilon}(\mathrm{He} \mathrm{I})$ is lower than $T_{\epsilon}(\mathrm{BJ})$, then the chemical abundances in the nebulae have two different abundance patterns. Otherwise, the abundance discrepancy between the CELs and the RLs is caused by temperature fluctuations within the nebula.

We attempted to explain the discrepancies in $\mathrm{C}$ and $\mathrm{O}$ abundance by including temperature fluctuations in the nebula. Peimbert et al. (1993) extended this effect to account for RL and $\mathrm{CEL} \mathrm{O}^{2+}$ abundance discrepancies by introducing the mean electron temperature $T_{0}$ and the electron temperature fluctuation parameter $t^{2}$, as follows,

$$
\begin{aligned}
& T_{0}\left(\mathrm{X}^{i+}\right)=\frac{\int T_{\epsilon} n_{\epsilon} N\left(\mathrm{X}^{i+}\right) d V}{\int n_{\epsilon} N\left(\mathrm{X}^{i+}\right) d V}, \\
& t^{2}=\frac{\int\left(T_{\epsilon}-T_{0}\right)^{2} n_{\epsilon} N\left(\mathrm{X}^{i+}\right) d V}{T_{0} \int n_{\epsilon} N\left(\mathrm{X}^{i+}\right) d V} .
\end{aligned}
$$

Using this temperature fluctuation model, we attempted to explain the discrepancy in $\mathrm{C}^{2+, 3+}$ and $\mathrm{O}^{2+}$ abundance.

Following the methods of Peimbert \& Torres-Peimbert (1971) and Peimbert (2003), we estimated the mean electron temperatures and $t^{2}$. When $t^{2} \ll 1$, the observed $T_{\epsilon}$ ([O III]), $T_{\epsilon}([\mathrm{N} I I])$, and $T_{\epsilon}(\mathrm{BJ})$ are written as follows,

$$
\begin{aligned}
& T_{\epsilon}([\mathrm{OIII}])=T_{0, h}\left[1+\frac{1}{2}\left(\frac{91300}{T_{0, h}}-3\right) t_{h}^{2}\right], \\
& T_{\epsilon}([\mathrm{NII}])=T_{0, l}\left[1+\frac{1}{2}\left(\frac{69070}{T_{0, l}}-3\right) t_{l}^{2}\right], \\
& T_{\epsilon}(\mathrm{BJ})=T_{0}\left(1-1.67 t^{2}\right),
\end{aligned}
$$

where $T_{0, h} \& t_{h}^{2}$ and $T_{0, h} \& t_{l}^{2}$ are the average temperatures and temperature fluctuation parameters in high- and lowionization zones, respectively; $\mathrm{C}^{+}, \mathrm{N}^{+}, \mathrm{O}^{+}$, and $\mathrm{S}^{+}$are in low- 
TABLE 9

The Elemental Abundances From CEL and RLs.

\begin{tabular}{|c|c|c|c|c|c|c|}
\hline $\mathrm{X}$ & $\begin{array}{c}\text { Types of } \\
\text { Emissions }\end{array}$ & $\mathrm{X} / \mathrm{H}$ & $\log (\mathrm{X} / \mathrm{H})+12$ & {$[\mathrm{X} / \mathrm{H}]$} & $\log \left(\mathrm{X}_{\odot} / \mathrm{H}\right)+12$ & $\operatorname{ICF}(X)$ \\
\hline $\mathrm{He}$ & RL & $1.08(-2) \pm 3.78(-2)$ & $11.04 \pm 0.15$ & $+0.11 \pm 0.15$ & $10.93 \pm 0.01$ & 1.00 \\
\hline $\mathrm{C}$ & RL & $2.33(-3) \pm 6.91(-4)$ & $9.37 \pm 0.13$ & $+0.98 \pm 0.13$ & $8.39 \pm 0.04$ & $1.44 \pm 0.38$ \\
\hline $\mathrm{C}$ & CEL & $1.04(-3) \pm 4.42(-4)$ & $9.02 \pm 0.18$ & $+0.63 \pm 0.19$ & $8.39 \pm 0.04$ & $1.01 \pm 0.42$ \\
\hline $\mathrm{N}$ & CEL & $3.85(-5) \pm 3.56(-6)$ & $7.59 \pm 0.04$ & $-0.24 \pm 0.06$ & $7.83 \pm 0.05$ & $3.28 \pm 0.26$ \\
\hline $\mathrm{O}$ & $\mathrm{RL}$ & $2.63(-4) \pm 7.54(-5)$ & $8.42 \pm 0.12$ & $-0.27 \pm 0.13$ & $8.69 \pm 0.05$ & $1.55 \pm 0.32$ \\
\hline $\mathrm{O}$ & CEL & $1.50(-4) \pm 6.09(-6)$ & $8.18 \pm 0.02$ & $-0.51 \pm 0.05$ & $8.69 \pm 0.05$ & 1.00 \\
\hline $\mathrm{Ne}$ & CEL & $2.67(-6) \pm 5.87(-7)$ & $6.43 \pm 0.10$ & $-1.44 \pm 0.14$ & $7.87 \pm 0.10$ & $1.05 \pm 0.22$ \\
\hline $\mathrm{S}$ & CEL & $1.36(-7) \pm 1.01(-8)$ & $5.13 \pm 0.03$ & $-2.06 \pm 0.05$ & $7.19 \pm 0.04$ & 1.00 \\
\hline $\mathrm{Cl}$ & CEL & $7.57(-9) \pm 2.24(-9)$ & $3.88 \pm 0.13$ & $-1.62 \pm 0.33$ & $5.50 \pm 0.30$ & $2.07 \pm 0.56$ \\
\hline $\mathrm{Ar}$ & CEL & $3.63(-8) \pm 9.65(-9)$ & $4.56 \pm 0.12$ & $-1.99 \pm 0.14$ & $6.55 \pm 0.08$ & $1.45 \pm 0.38$ \\
\hline $\mathrm{Xe}$ & CEL & $>5.05(-10)$ & $>2.75$ & $>+0.48$ & $2.27 \pm 0.02$ & $1.94 \pm 0.51$ \\
\hline $\mathrm{Ba}$ & CEL & $<3.23(-10)$ & $<2.51$ & $<+0.33$ & $2.18 \pm 0.03$ & 1 \\
\hline
\end{tabular}

TABLE 10

ELEMENTAL ABUNDANCES DERIVED BY PREVIOUS WORKS AND BY THIS WORK.

\begin{tabular}{|c|c|c|c|c|c|c|c|c|c|c|}
\hline References & $\mathrm{He}$ & $\mathrm{C}$ & $\mathrm{N}$ & $\mathrm{O}$ & $\mathrm{Ne}$ & $\mathrm{S}$ & $\mathrm{Cl}$ & $\mathrm{Ar}$ & $\mathrm{Xe}$ & $\mathrm{Ba}$ \\
\hline This work (RL) & 11.04 & 9.37 & $\ldots$ & 8.42 & $\ldots$ & $\ldots$ & $\ldots$ & $\ldots$ & $\cdots$ & $\cdots$ \\
\hline This work (CEL) & $\cdots$ & 9.02 & 7.56 & 8.18 & 6.43 & 5.13 & 3.88 & 4.56 & $>2.75$ & $<\mathbf{2 . 5 1}$ \\
\hline Kwitter et al. (2003) & 11.08 & $\cdots$ & 7.76 & 8.30 & 6.60 & 5.30 & $\cdots$ & 4.30 & $\cdots$ & $\cdots$ \\
\hline Henry et al. $(1996)^{a}$ & 11.00 & 8.68 & 7.76 & 8.40 & 6.44 & $\cdots$ & $\cdots$ & $\cdots$ & $\cdots$ & $\cdots$ \\
\hline Torres-Peimbert \& Peimbert $\left(1979, t^{2}=0\right)$ & 10.99 & $9.39^{b}$ & 7.75 & 8.37 & 6.68 & $\cdots$ & $\ldots$ & $\ldots$ & $\cdots$ & $\ldots$ \\
\hline Torres-Peimbert \& Peimbert $\left(1979, t^{2}=0.035\right)$ & 10.99 & $9.39^{b}$ & 7.87 & 8.50 & 6.80 & $\cdots$ & $\cdots$ & $\cdots$ & $\cdots$ & $\cdots$ \\
\hline
\end{tabular}

Note. - The CEL abundances with $t^{2} \neq 0$ by us are listed in Table 12

${ }^{a}$ Derived from photo-ionization modeling.

b Derived from C II $\lambda 4267 \AA$ line.

TABLE 11

C, AR, Xe, AND Ba ABUndances in PNe.

\begin{tabular}{lllllll}
\hline \hline Nebulae & {$[\mathrm{C} / \mathrm{Ar}]$} & {$[\mathrm{Xe} / \mathrm{Ar}]$} & {$[\mathrm{Ba} / \mathrm{Ar}]$} & {$[\mathrm{Ba} / \mathrm{Xe}]$} & {$[\mathrm{Ar} / \mathrm{H}]$} & \multicolumn{1}{c}{ Ref. } \\
\hline IC418 & +0.94 & +0.94 & +0.33 & -0.61 & -0.54 & $(1),(2)$ \\
IC2501 & +0.66 & -0.03 & -0.06 & +0.09 & -0.27 & $(1),(3),(4)$ \\
IC4191 & +0.93 & +0.50 & +0.83 & +0.33 & -0.51 & $(1),(5),(6)$ \\
M3-15 & +0.23 & +1.15 & $\ldots$ & $\ldots$ & -0.25 & $(7),(8)$ \\
NGC2440 & +0.71 & -0.36 & +0.79 & +1.15 & -0.24 & $(1),(9)$ \\
NGC5189 & -0.10 & -0.08 & $\ldots$ & $\ldots$ & -0.06 & $(7),(8)$ \\
NGC7027 & +0.91 & +0.93 & +0.43 & -0.50 & -0.20 & $(1),(10)$ \\
PC14 & +0.43 & +0.13 & $\ldots$ & $\ldots$ & -0.17 & $(7),(8)$ \\
Pe1-1 & +0.74 & +1.00 & $\ldots$ & $\ldots$ & -0.23 & $(7),(8)$ \\
BoBn1 & +2.85 & $<+1.95$ & $>+2.34$ & $>+0.38$ & -2.22 & $(11)$ \\
H4-1 & +2.62 & $>+2.47$ & $<+2.32$ & $<-0.18$ & -1.99 & $(7)$ \\
\hline
\end{tabular}

REFERENCES. - (1) Sharpee et al. (2007); (2) Pottasch et al. (2004); (3) Henry et al. (2004); (4) Rola \& Stasińska (1994); (5) Pottasch et al. (2005); (6) Tsamis et al. (2004); (7) This work; (8) García-Rojas et al. (2012); (9) Bernard Salas et al. (2002); (10) Zhang et al. (2005b); (11) Otsuka et al. (2010)

Note. - The abundances are estimated from CELs, except for C in IC4191 which is estimated using recombination lines.

ionization zones and the other elements are in high-ionization zones (See Table 8).

Based on the assumption that $t^{2}=t_{h}^{2}=t_{l}^{2}$, we found that $T_{0, h}=12590 \pm 340 \mathrm{~K}, T_{0, l}=10760 \pm 290 \mathrm{~K}, T_{0}=12590 \pm 1100 \mathrm{~K}$, and $t^{2}=0.030 \pm 0.007$ minimize the ADFs in $\mathrm{C}^{2+, 3+}$ and $\mathrm{O}^{2+}$ using the combination of equations (4)-(6). Our determined $t^{2}$ is in agreement with Torres-Peimbert \& Peimbert (1979), who determined $t^{2}=0.035$.

Next, the average line-emitting temperatures for each line at $\lambda, T_{\epsilon}(\lambda)$, and $T_{\epsilon}(\mathrm{H} \beta)$ can be written by following the method
TABLE 12

The Ionic And Elemental AbUNDANCES From CELs in $t^{2}=0.03$.

\begin{tabular}{lclc}
\hline \hline $\mathrm{X}^{\mathrm{m}+}$ & $\left(\mathrm{X}^{\mathrm{m}+} / \mathrm{H}^{+}\right)_{t^{2} \neq 0}$ & $\mathrm{X}^{\mathrm{m}+}$ & $\left(\mathrm{X}^{\mathrm{m}+} / \mathrm{H}^{+}\right)_{t^{2}} \neq 0$ \\
\hline $\mathrm{C}^{+}$ & $3.98(-4) \pm 5.06(-5)$ & $\mathrm{Ne}^{2+}$ & $2.42(-6) \pm 1.91(-7)$ \\
$\mathrm{C}^{2+}$ & $6.25(-4) \pm 9.38(-5)$ & $\mathrm{S}^{+}$ & $2.27(-8) \pm 1.37(-9)$ \\
$\mathrm{C}^{3+}$ & $8.96(-5) \pm 1.85(-5)$ & $\mathrm{S}^{2+}$ & $8.63(-8) \pm 9.79(-9)$ \\
$\mathrm{N}^{+}$ & $1.21(-5) \pm 5.77(-7)$ & $\mathrm{S}^{3+}$ & $3.48(-8) \pm 4.09(-9)$ \\
$\mathrm{O}^{+}$ & $4.83(-5) \pm 3.78(-6)$ & $\mathrm{Cl}^{2+}$ & $4.37(-9) \pm 5.21(-10)$ \\
$\mathrm{O}^{2+}$ & $1.17(-4) \pm 6.82(-6)$ & $\mathrm{Ar}^{2+}$ & $2.05(-8) \pm 1.12(-9)$ \\
$\mathrm{O}^{3+}$ & $7.80(-6) \pm 7.23(-7)$ & $\mathrm{Ar}^{3+}$ & $9.25(-9) \pm 9.58(-10)$ \\
$\mathrm{Ne}^{+}$ & $4.66(-7) \pm 6.81(-8)$ & $\mathrm{Xe}^{2+}$ & $>3.61(-10)$ \\
\hline \hline $\mathrm{X}$ & $\log (\mathrm{X} / \mathrm{H})_{t^{2}}{ }^{2+}+12$ & $\mathrm{X}$ & $\log (\mathrm{X} / \mathrm{H})_{t^{2}}{ }^{2+}+12$ \\
\hline $\mathrm{C}$ & $9.05 \pm 0.18$ & $\mathrm{~S}$ & $5.15 \pm 0.03$ \\
$\mathrm{~N}$ & $7.64 \pm 0.04$ & $\mathrm{Cl}$ & $3.95 \pm 0.13$ \\
$\mathrm{O}$ & $8.24 \pm 0.02$ & $\mathrm{Ar}$ & $4.62 \pm 0.11$ \\
$\mathrm{Ne}$ & $6.48 \pm 0.10$ & $\mathrm{Xe}$ & $>2.84$ \\
\hline
\end{tabular}

Note. - The RL elemental $\mathrm{C}$ and $\mathrm{O}$ abundances in $t^{2}=0.03$ are $9.35 \pm 0.13$ and $8.40 \pm 0.12$, respectively (See text in detail).

of Peimbert et al. (2004):

$$
\begin{aligned}
& T_{\epsilon}(\lambda)=T_{0, h, l} \\
& \times\left\{1+\left[\frac{\left(\Delta E / k T_{0, h, l}\right)^{2}-3\left(\Delta E / k T_{0, h, l}\right)+0.75}{\left(\Delta E / k T_{0, h, l}\right)-0.5}\right] \frac{t^{2}}{2}\right\}, \\
& T_{\epsilon}(\mathrm{H} \beta)=T_{0, h, l}\left(1-\frac{1.633}{2} t^{2}\right),
\end{aligned}
$$

where $T_{0, h, l}$ is $12590 \pm 340 \mathrm{~K}$ for the elements in high ionization zone and is $10760 \pm 290 \mathrm{~K}$ for those in low ionization zone, the $\Delta E$ is the difference energy between the upper and lower level of of the target lines, $k$ is Boltzmann constant, 
TABLE 13

COMPARISON OF THE OBSERVED ABUNDANCES WITH THE MODEL PREDICTION.

\begin{tabular}{lrrrrrrrr}
\hline \hline \multicolumn{1}{c}{ References } & $\mathrm{C}$ & $\mathrm{N}$ & $\mathrm{O}$ & $\mathrm{Ne}$ & $\mathrm{S}$ & $\mathrm{Ar}$ & $\mathrm{Xe}$ & $\mathrm{Ba}$ \\
\hline $0.9 M_{\odot}$ & 9.04 & 7.57 & 7.63 & 7.87 & 5.00 & 4.28 & 1.56 & 2.37 \\
initial abundances & 6.28 & 5.68 & 6.54 & 5.78 & 4.97 & 4.25 & 0.01 & 0.00 \\
\hline $2.0 M_{\odot}$ & 9.55 & 6.84 & 7.93 & 8.66 & 5.34 & 4.57 & 2.11 & 2.52 \\
$+r+2.0$ dex & & & & & & & & \\
initial abundances & 6.06 & 4.64 & 6.82 & 6.06 & 5.26 & 4.49 & 1.90 & 1.29 \\
\hline This work $\left(t^{2}=0\right)$ & 9.02 & 7.56 & 8.18 & 6.43 & 5.13 & 4.56 & $>2.75$ & $<2.51$ \\
\hline
\end{tabular}

respectively. Finally, the ionic abundances can be estimated by following the method of Peimbert et al. (2004), which accounts for the effects of fluctuations in temperature:

$$
\begin{aligned}
& \frac{N\left(\mathrm{X}^{m+}\right)_{t^{2} \neq 0.00}}{N\left(\mathrm{X}^{m+}\right)_{t^{2}=0.00}}=\frac{T_{\epsilon}(\mathrm{H} \beta)^{-0.87} T_{\epsilon}(\lambda)^{0.5}}{T_{\epsilon}([\mathrm{OIII}],[\mathrm{NII}])^{-0.37}} \\
& \times \exp \left[\frac{\Delta E}{k T_{\epsilon}(\lambda)}-\frac{\Delta E}{k T_{\epsilon}([\mathrm{OIII}],[\mathrm{NII}])}\right] .
\end{aligned}
$$

The resulting CEL ionic and elemental abundances are summarized in Table 12. The ADFs are slightly improved for $\mathrm{C}^{2+}$ and $\mathrm{O}^{2+}$, with values of $1.94 \pm 0.45$ and $1.45 \pm 0.30$, respectively, whereas the $\mathrm{ADF}$ for $\mathrm{C}^{3+}$ remains high, with a value of $4.31 \pm 1.03$. Because most of the ionic $\mathrm{C}$ abundances are in a doubly ionized stage, and the CEL $\mathrm{C}^{2+}$ abundance approaches that of the $\mathrm{RL} \mathrm{C}^{2+}$, the elemental CEL $\mathrm{C}$ abundance is very close to the RL C abundance (9.35 dex for the RL and 9.05 dex for the CEL), resolving the $\mathrm{C}$ abundance discrepancy. The elemental $\mathrm{O}$ abundances agree within error, 8.40 for the RL and 8.24 for the CEL.

Two difference abundance patterns could cause the ionic $\mathrm{C}^{2+, 3+}$ and $\mathrm{O}^{2+}$ abundances. However, even if these abundance patterns appear in $\mathrm{H} 4-1$, the difference between these ionic abundances would be negligible.

\subsection{Comparison with theoretical model}

The large enhancement of the Xe abundance is the most remarkable finding for H4-1. In this section, we compare the observed abundances with the nucleosynthesis models for the initially $0.9 M_{\odot}$ and $2.0 M_{\odot}$ single stars with $[\mathrm{X} / \mathrm{H}]=$ 2.19 from Lugaro et al. (2012), and we verify whether the observed Xe abundance could be explained using their models. From the galaxy chemical evolution model of Kobayashi et al. (2011), an $[\mathrm{Fe} / \mathrm{H}]$ ratio of about -2.30 in $\mathrm{H} 4-1$ is estimated using the relation $[\mathrm{Fe} / \mathrm{H}]=[\mathrm{Ar} / \mathrm{H}]-0.30$. Therefore, the results of Lugaro et al. (2012) are good comparisons.

For the initially $0.9 M_{\odot}$ star model, Lugaro et al. (2012) adopted scaled-solar abundances as the initial composition for all elements from $\mathrm{C}$ to $\mathrm{Pb}$. Both the initial $\mathrm{Xe}$ and $\mathrm{Ba}$ abundances are $\sim 0$, meaning that the final predicted abundances of these two elements are pure products of the $s$-process in their progenitors. In the $2.0 M_{\odot}$ model, they initially adopted the $[r / \mathrm{Fe}]=+2$. In Table 13 , we compare our results with the predictions by Lugaro et al. (2012). The initial abundances are also listed. The models of Lugaro et al. (2012) include a partial mixing zone $(\mathrm{PMZ})$ of $2(-3) M_{\odot}$ that produces a ${ }^{13} \mathrm{C}$ pocket during the interpulse period and releases extra free neutrons $(n)$ through ${ }^{13} \mathrm{C}(\alpha, n){ }^{16} \mathrm{O}$ to obtain $n$-process elements. The $0.9 M_{\odot}$ and the $2.0 M_{\odot}$ star models predict that the respective stars experienced 38 and 27 times thermal pulses and the occurrence of a TDU.

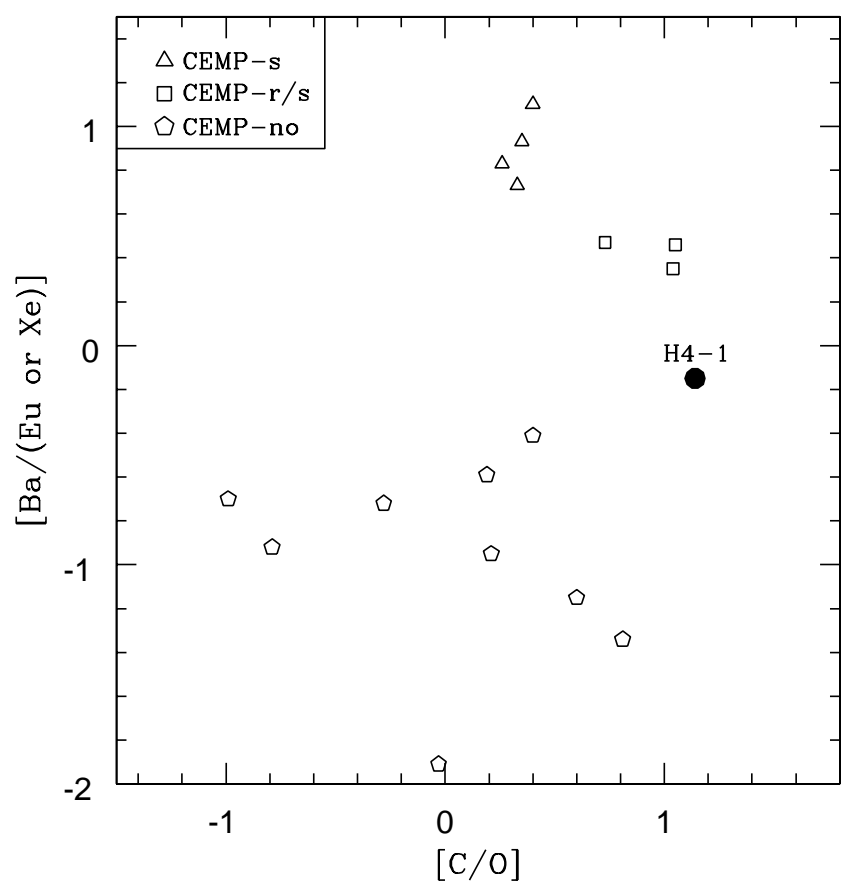

FIG. 5.- The $[\mathrm{Ba} /(\mathrm{Eu}$ or $\mathrm{Xe})]$ versus [C/O] diagram. For H4-1, we used the $[\mathrm{Ba} / \mathrm{Xe}]$ abundance. The $[\mathrm{Ba} / \mathrm{Xe}]$ abundance of $\mathrm{H} 4-1$ is the upper limit. We used the abundances of CEMP stars provided by Suda et al. (2011). CEMP stars are classified as the criteria summarized in Table 14 The $[\mathrm{Fe} / \mathrm{H}]$ of CEMP stars plotted in this diagram is from -4.75 to -2.03 in CEMP-no, from -2.7 to -2.2 in CEMP-s, and from -2.72 to -2.03 in CEMP-r/s, respectively.

The $0.9 M_{\odot}$ model gives a good agreement with the observed $\mathrm{C}$ and $\mathrm{N}$ abundances, however, there are discrepancies between the observed and the model predicted $\alpha$-elements. The production of $\mathrm{Xe}$ is mainly a result of the $r$-process; Bisterzo et al. (2011) reported that the solar Xe and Eu are mainly from the $r$-process ( $84.6 \%$ and $94 \%$, respectively), although the contribution from the $r$-process to the Xe is disputable. The $0.9 M_{\odot}$ model predicted the $\mathrm{Xe} / \mathrm{H}$ abundance of 3.63(-11), while the observed abundance in $\mathrm{H} 4-1$ is $5.62(-$ 10). Therefore, most the xenon in H4-1 is produced by the $r$-process in primordial SNe.

If the progenitor of H4-1 was formed in an $r$-process rich environment, the observed $\alpha$ elements and Xe could be explained. Compared to the $0.9 M_{\odot}$ model, except for $\mathrm{N}$ and $\mathrm{Ne}$, the $r$-process enhanced model for $2.0 M_{\odot}$ stars explains the observed $\mathrm{O}, \mathrm{S}, \mathrm{Ar}$, and Xe abundances well. About 0.2-0.3 dex of the observed $\alpha$-elements are SN products.

Through comparison with the theoretical models, we suppose that the observed $\mathrm{Xe}$ is mostly synthesized by $r$-process in SNe. The observed abundances could be explained by the model for $2.0 M_{\odot}$ stars with the initial $[r / F e]=+2.0$.

\subsection{Comparison with CEMP stars and the evolution and evolution of $\mathrm{H} 4-1$}

Although the models by Lugaro et al. (2012) explain the observed abundances, it is difficult to explain the evolutional time scale of H4-1 with a $2.0 M_{\odot}$ single-star evolution models, because such mass stars cannot survive in the Galactic halo up to now.

Therefore, it is possible that H4-1 evolved from a binary, similar to the evolution of CEMP stars, and its progenitor was polluted by SNe. Our definition of CEMP stars is summarized in Table 14, following Beers \& Christlieb (2005). CEMP-s 
TABLE 14

CLASSIFICATION OF CEMP STARS IN THIS WORK.

\begin{tabular}{ll}
\hline \hline \multicolumn{1}{c}{ Class } & Criteria \\
\hline CEMP- $s$ & {$[\mathrm{Fe} / \mathrm{H}] \leq-2,[\mathrm{C} / \mathrm{Fe}]>+1.0,[\mathrm{Ba} / \mathrm{Fe}]>+1.0,[\mathrm{Ba} / \mathrm{Eu}]>+0.5$} \\
CEMP-r $/ s$ & {$[\mathrm{Fe} / \mathrm{H}] \leq-2,[\mathrm{C} / \mathrm{Fe}]>+1.0,[\mathrm{Ba} / \mathrm{Fe}]>+1,0<[\mathrm{Ba} / \mathrm{Eu}]<+0.5$} \\
CEMP-no & {$[\mathrm{Fe} / \mathrm{H}] \leq-2,[\mathrm{C} / \mathrm{Fe}]>+1.0,[\mathrm{Ba} / \mathrm{Fe}]<0.0$} \\
\hline
\end{tabular}

Note. - In H4-1, the expected $[\mathrm{Fe} / \mathrm{H}]$ and $[\mathrm{Ba} / \mathrm{Fe}]$ are -2.3 and $<+2.6$ and the observed $[\mathrm{C} / \mathrm{Fe}]$ and $[\mathrm{Ba} / \mathrm{Xe}]$ are +2.83 and $<-0.15$, respectively.

stars are $s$-process rich, CEMP- $r / s$ are both $s$ - and $r$-process rich. The CEMP stars which are neither CEMP-s nor CEMP$r / s$ are classified as the CEMP-no. Since the expected $[\mathrm{Fe} / \mathrm{H}]$ and $[\mathrm{Ba} / \mathrm{Fe}]$ are -2.3 and $<+2.6$ and the observed $[\mathrm{C} / \mathrm{Fe}]$ and $[\mathrm{Ba} / \mathrm{Xe}]$ are +2.83 and $<-0.15$, respectively, $\mathrm{H} 4-1$ can be classified as CEMP-r/s or CEMP-no.

In Fig. 5] we present the $[\mathrm{Ba} /(\mathrm{Eu}$ or $\mathrm{Xe})]$ versus $[\mathrm{C} / \mathrm{O}] \mathrm{di}-$ agram between H4-1 and CEMP stars. The data of CEMP stars are taken from Suda et al. (2011) and the classification is based on Table 14 The metallicities of CEMP- $s$ and CEMP-r/s stars are widely spread, while those of CEMP-no are mostly $\lesssim-3$ (e.g., Aoki et al. 2007). Fig. 5 indicates that H4-1 is similar to CEMP-r/s and CEMP-no, assuming that the $[\mathrm{Eu} / \mathrm{H}]$ abundance in $\mathrm{H} 4-1$ is similar to its $[\mathrm{Xe} / \mathrm{H}]$ abundance.

Although H4-1 has a similar origin and evolution as CEMP$r / s$ and CEMP-no stars, the origin of these CEMP stars is a topic of much debate (Lugaro et al. 2012; Bisterzo et al. 2011; Jonsell et al.2006; Ito et al.2013; Zijlstra 2004). As proposed by Zijlstra (2004) to explain the abundances of $r / s$-rich stars, the progenitor of H4-1 might be a binary composing of e.g, $\sim 0.8-0.9 M_{\odot}$ and $\sim 5 M_{\odot}$ (from their Fig. 2), which might evolve into a SN. However, there are problems on the $\mathrm{N}$ and Xe productions in such massive primary scenario for explanation of H4-1's evolution. For instance, the $5 M_{\odot}$ with $r+0.4$ dex models by Lugaro et al. (2012) predicted highly enhanced $\mathrm{N}$ (9.11) by the hot bottom burning and low Xe abundances (0.99 dex). While, Suda et al. (2013) argued that the inclusion of mass-loss suppression in metal-poor AGB stars can inhibit such N-enhanced metal-poor stars. Therefore, at the present, we think that the primary star should have never experienced the hot bottom burning.

If bipolar nebulae are created by stable mass-transfer during Roche lobe overflow, the initial mass ratio of the primary to secondary is $\sim 1-2$, according to Phillips (2000). If this is the case for the bipolar nebula formation in $\mathrm{H} 4-1$ and the secondary is $\sim 0.8-0.9 M_{\odot}$, the primary star would be $\sim 0.8-1.8$ $M_{\odot}$. For example, Otsuka et al. (2010) explain the observed chemical abundances of BoBn1 using the binary model composed of $0.75 M_{\odot}+1.5 M_{\odot}$ stars.

\section{SUMMARY}

We analyzed the multi-wavelength spectra of the halo PN H4-1 from Subaru/HDS, GALEX, SDSS, and Spitzer/IRS in order to to determine chemical abundances, in particular, $n$ capture elements, solve the $\mathrm{C}$ abundance discrepancy problem, and obtain insights on the origin and evolution of H4-1. We determined the abundances of 10 elements based on the over 160 lines detected in those data. The $\mathrm{C}$ and $\mathrm{O}$ abundances were derived from both CELs and RLs. We found the discrepancies between the CEL and the RL abundances of $\mathrm{C}$ and $\mathrm{O}$, respectively and they can be explained by considering temperature fluctuation effect. The large discrepancy in the $\mathrm{C}$ abundance between CEL and RL in H4-1 was solved by our study. In HDS spectrum, we detected the [Xe III] $\lambda 5846 \AA$ line in H4-1 for the first time. H4-1 is the most Xe enhanced PN among the Xe detected PNe. The observed abundances can be explained by a $\sim 2.0 M_{\odot}$ single star model with initially $[r / \mathrm{Fe}]=+2.0$ of Lugaro et al. (2012). The observed Xe abundance would be a product of the $r$-process in primordial SNe. About 0.2-0.3 dex of the $\alpha$ elements are also the products by these $\mathrm{SNe}$. The $[\mathrm{C} / \mathrm{O}]-[\mathrm{Ba} /(\mathrm{Eu}$ or $\mathrm{Xe})]$ diagram suggests that the progenitor of H4-1 shares the evolution with CEMP-r/s and CEMP-no stars. The progenitor of H4-1 is a presumably binary formed in an $r$-process rich environment.

\section{ACKNOWLEDGMENTS}

We are grateful to the anonymous referee for a careful reading and valuable suggestions. We sincerely thank Takuma Suda and Siek Hyung for fruitful discussions on low-mass AGB nucleosynthesis and PN abundances. This work is mainly based on data collected at the Subaru Telescope, which is operated by the National Astronomical Observatory of Japan (NAOJ). This work is in part based on GALEX archive data downloaded from the MAST. This work is in part based on archival data obtained with the Spitzer Space Telescope, which is operated by the Jet Propulsion Laboratory, California Institute of Technology under a contract with NASA. Support for this work was provided by an award issued by JPL/Caltech. Funding for the SDSS has been provided by the Alfred P. Sloan Foundation, the Participating Institutions, the National Aeronautics and Space Administration, the National Science Foundation, the U.S. Department of Energy, the Japanese Monbukagakusho, the Max Planck Society, and the Higher Education Funding Council for England.

\section{REFERENCES}

Aoki W., Beers T.C., Christlieb N., et al., 2007, ApJ 655, 492

Asplund M., Grevesse N., Sauval A.J., Scott P., 2009, ARA\&A 47, 481

Beers T.C., Christlieb N., 2005, ARA\&A 43, 531

Benjamin R.A., Skillman E.D., Smits D.P., 1999, ApJ 514, 307

Bernard Salas J., Pottasch S.R., Feibelman W.A., Wesselius P.R., 2002, A\&A 387, 301

Biemont E., Hansen J.E., Quinet P., Zeippen C.J., 1995, A\&AS 111, 333

Bisterzo S., Gallino R., Straniero O., Cristallo S., Käppeler F., 2011, MNRAS 418, 284

Boothroyd A.I., Sackmann I.J., 1988, ApJ 328, 671

Cardelli J.A., Clayton G.C., Mathis J.S., 1989, ApJ 345, 245

Dinerstein H.L., Richter M.J., Lacy J.H., Sellgren K., 2003, AJ 125, 265

Ferland G.J., Korista K.T., Verner D.A., et al., 1998, PASP 110, 761

Fluks M.A., Plez B., The P.S., et al., 1994, A\&AS 105, 311

Fujimoto M.Y., Ikeda Y., Iben, Jr. I., 2000, ApJ 529, L25
García-Rojas J., Peña M., Morisset C., Mesa-Delgado A., Ruiz M.T., 2012, A\&A 538, A54

García-Rojas J., Peña M., Peimbert A., 2009, A\&A 496, 139

Henry R.B.C., Kwitter K.B., Balick B., 2004, AJ 127, 2284

Henry R.B.C., Kwitter K.B., Howard J.W., 1996, ApJ 458, 215

Higdon S.J.U., Devost D., Higdon J.L., et al., 2004, PASP 116, 975

Houck J.R., Roellig T.L., van Cleve J., et al., 2004, ApJS 154, 18

Ito H., Aoki W., Beers T.C., et al., 2013, ApJ 773, 33

Jonsell K., Barklem P.S., Gustafsson B., et al., 2006, A\&A 451, 651

Klose J.Z., Fuhr J.R., Wiese W.L., 2002, Journal of Physical and Chemical

Reference Data 31, 217

Kobayashi C., Karakas A.I., Umeda H., 2011, MNRAS 414, 3231

Kwitter K.B., Henry R.B.C., Milingo J.B., 2003, PASP 115, 80

Lattanzio J.C., 1987, ApJ 313, L15 
Liu X.W., 2006, In: Barlow M.J., Méndez R.H. (eds.), Planetary Nebulae in our Galaxy and Beyond, vol. 234 of IAU Symposium, pp. 219-226

Liu X.W., Luo S.G., Barlow M.J., Danziger I.J., Storey P.J., 2001, MNRAS 327,141

Liu X.W., Storey P.J., Barlow M.J., et al., 2000, MNRAS 312, 585

Lodders K., 2003, ApJ 591, 1220

Lugaro M., Karakas A.I., Stancliffe R.J., Rijs C., 2012, ApJ 747, 2

Mal'Kov Y.F., 1997, Astronomy Reports 41, 760

Martin D.C., Fanson J., Schiminovich D., et al., 2005, ApJ 619, L1

Noguchi K., Aoki W., Kawanomoto S., et al., 2002, PASJ 54, 855

Otsuka M., Izumiura H., Tajitsu A., Hyung S., 2008a, ApJ 682, L105

Otsuka M., Izumiura H., Tajitsu A., Hyung S., 2008b, In: Suda T., Nozawa T., Ohnishi A., et al. (eds.), Origin of Matter and Evolution of Galaxies, vol. 1016 of American Institute of Physics Conference Series, pp. $427-429$

Otsuka M., Tajitsu A., Hyung S., Izumiura H., 2010, ApJ 723, 658

Peimbert A., 2003, ApJ 584, 735

Peimbert A., Peimbert M., Ruiz M.T., 2005, ApJ 634, 1056

Peimbert M., Peimbert A., Ruiz M.T., Esteban C., 2004, ApJS 150, 431

Peimbert M., Storey P.J., Torres-Peimbert S., 1993, ApJ 414, 626

Peimbert M., Torres-Peimbert S., 1971, Boletin de los Observatorios Tonantzintla y Tacubaya 6, 21

Phillips J.P., 2000, AJ 119, 342

Pottasch S.R., Beintema D.A., Feibelman W.A., 2005, A\&A 436, 953

Pottasch S.R., Bernard-Salas J., Beintema D.A., Feibelman W.A., 2004, A\&A 423, 593
Rauch T., Heber U., Werner K., 2002, A\&A 381, 1007

Rola C., Stasińska G., 1994, A\&A 282, 199

Ruiz M.T., Peimbert A., Peimbert M., Esteban C., 2003, ApJ 595, 247

Schoening T., Butler K., 1998, A\&AS 128, 581

Sharpee B., Zhang Y., Williams R., et al., 2007, ApJ 659, 1265

Sterling N.C., Dinerstein H.L., Hwang S., et al., 2009, PASA 26, 339

Storey P.J., Hummer D.G., 1995, MNRAS 272, 41

Suda T., Komiya Y., Yamada S., et al., 2013, MNRAS 432, L46

Suda T., Yamada S., Katsuta Y., et al., 2011, MNRAS 412, 843

Tajitsu A., Otsuka M., 2004, In: Meixner M., Kastner J.H., Balick B., Soker N. (eds.), Asymmetrical Planetary Nebulae III: Winds, Structure and the Thunderbird, vol. 313 of Astronomical Society of the Pacific Conference Series, p. 202

Torres-Peimbert S., Peimbert M., 1979, RMxAA 4, 341

Tsamis Y.G., Barlow M.J., Liu X.W., Storey P.J., Danziger I.J., 2004, MNRAS 353, 953

Vassiliadis E., Wood P.R., 1994, ApJS 92, 125

Zhang Y., Liu X.W., Liu Y., Rubin R.H., 2005a, MNRAS 358, 457

Zhang Y., Liu X.W., Luo S.G., Péquignot D., Barlow M.J., 2005b, A\&A 442, 249

Zijlstra A.A., 2004, MNRAS 348, L23 